\title{
DiVERSIDAD DEL GÉNERO QUERCUS (FAGACEAE) EN MÉXICO
}

\author{
Susana Valencia-A. \\ Herbario de la Facultad de Ciencias (FCME), Departamento de Biología Comparada, Universidad Nacional \\ Autónoma de México, Ciudad Universitaria, México, 04510, México D.F. Correo-e: sva@hp.fciencias.unam.mx
}

\begin{abstract}
Resumen: Se presenta una lista preliminar de 161 especies del género Quercus para México, ubicadas en tres secciones: 76 en la sección Lobatae (encinos rojos), 81 en la sección Quercus (encinos blancos) y cuatro especies en la sección Protobalanus (encinos intermedios). Se calcula que 109 especies son endémicas del país, de las cuales 47 pertenecen a la sección Quercus, 61 a la sección Lobatae y una a Protobalanus. México comparte con Estados Unidos 33 especies del género, mientras que con Centroamérica comparte 20. Los estados con mayor diversidad de especies son Oaxaca, Nuevo León, Jalisco, Chihuahua y Veracruz. Las especies con distribución más amplia en nuestro país son $Q$. candicans, $Q$. castanea, $Q$. crassifolia, $Q$. laeta, $Q$. microphylla, $Q$. obtusata y $Q$. rugosa. Altitudinalmente las especies de Quercus se desarrollan entre 0 y 3,500 m, pero son más frecuentes entre 1,000 y 3,000 m. El conocimiento del género Quercus en México es aún deficiente y se necesita realizar más estudios en torno a este importante género.
\end{abstract}

Palabras clave: diversidad, encino, endemismo, México, Quercus.

\begin{abstract}
This study presents a preliminary list of 161 species from the genus Quercus, all native to Mexico. These species belong to three sections: section Lobatae (red oaks, 76 species), Quercus (white oaks, 81 species), and Protobalanus (intermediate oaks, 4 species). It is estimated that 109 are endemic to Mexico, 47 of which belong to section Quercus, 61 to section Lobatae, and 1 to section Protobalanus. Of all the oak species found in Mexico, 33 of them extend their range into the United States, while only 20 are also found in Central America. The states with the greatest oak divesity are Oaxaca, Nuevo León, Jalisco, Chihuahua and Veracruz. Species widely distributed in Mexico are $Q$. candicans, $Q$. castanea, $Q$. crassifolia, Q. laeta, Q. microphylla, Q. obtusata and $Q$. rugosa. In general, Mexican oak species grow in elevations ranging form 0 to 3,500 m; however, they are found predominantly between 1,000 and 3,000 m. Knowledge on the taxonomy of Mexican oak species remains incomplete; therefore, further research is needed around this important genus.
\end{abstract}

Keywords: diversity, endemism, Mexico, oak, Quercus.

E n la familia Fagaceae el género Quercus es el que presenta mayor distribución en todo el mundo. Se encuentra en casi todos los bosques templados del Hemisferio Norte, así como en algunas regiones tropicales y subtropicales del mismo. Incluso existen algunas especies en hábitats más secos, en el sureste de Asia y nororiente de África. En América se localiza desde Canadá hasta Colombia, incluyendo Cuba.

Se reconocen dos centros de diversidad para el género. El primero se localiza en el sureste de Asia con alrededor de 125 especies (Menitsky, 1984, en Govaerts y Frodin, 1998). El segundo se presenta en México, particularmente en las regiones montañosas, en donde forman parte importante de los bosques templados (Govaerts y Frodin, 1989).

La riqueza específica total para el género Quercus es

difícil de precisar. Algunos autores estiman que oscila alrededor de 300 (Lawrence, 1951) o 400 entidades (Nixon et al., 1997). Otros opinan que hay aproximadamente 500 (Manos et al., 1999) o 531 especies (Govaerts y Frodin, 1998). Los menos conservadores piensan que puede haber alrededor de 600 taxa específicos en todo el mundo (Soepadmo, 1972 en Jones, 1986).

El número de especies para México tampoco se conoce con exactitud. Trelease (1924) calcula que hay alrededor de 253 especies. En su obra monográfica, Camus (1934-1954) cita 238 especies. Más recientemente, estimaciones de Nixon (1993a) arrojan entre 135 y 150 especies. Otras cifras son la proporcionada por González (1993), quien considera que en México hay entre 173 y 213 especies del género Quercus, y las de Zavala-Chávez (1995), quien 
estimó que puede haber entre 125 y 150 especies de encinos. La revisión de Govaerts y Frodin (1998) indican la cifra de 251 especies de encinos en México.

Existe gran interés en torno al género Quercus atribuido a su alta diversidad y a su importancia ecológica y económica. Sin embargo, el conocimiento sobre su riqueza aún es deficiente. Diversos problemas dificultan conocer el número exacto de especies del género Quercus: la gran variación morfológica específica (aun dentro del mismo individuo), la escasez, carencia e inaccesibilidad del material tipo y de la literatura original, descripciones originales deficientes, la sobredescripción de muchas de las especies (Muller, 1942a; Muller y McVaugh, 1972) y la frecuente hibridación de las mismas (Hardin, 1975; Grant, 1989), todo ello aunado a los problemas que se tienen para visitar herbarios (nacionales e internacionales) y realizar trabajo de campo, así como a la diferencia de criterios de los especialistas del grupo.

La resolución de diversos problemas taxonómicos ha sido posible gracias al estudio particular de algunas especies mexicanas del género Quercus. Entre éstos se pueden mencionar los siguientes trabajos. Muller y McVaugh (1972) revisaron las especies descritas por Née y por Humboldt y Bonpland y propusieron sinónimos y nombres aceptados para cada una de ellas. Nixon y Muller (1992) estudiaron a la especie $Q$. laceyi Small para diferenciarla de $Q$. glaucoides M.Martens et Galeotti. Nixon y Muller (1993) trabajaron el complejo de $Q$. hypoxantha Trel. en el noreste de México. Spellenberg $(1995,1998)$ aclaró la naturaleza híbrida de dos supuestas especies de encinos. Spellenberg y Bacon (1996) trabajaron con algunas especies de la serie Racemiflorae. Romero et al. (2000) estudiaron a $Q$. acutifolia Née y a $Q$. conspersa Benth., resaltando los caracteres que las pueden diferenciar. Vázquez et al. (2004) estudiaron a $Q$. hirtiifolia Vázquez, Valencia et Nixon y analizan los caracteres de las especies similares a ésta. Valencia et al. (en proceso) están revisando la taxonomía de las especies del complejo de Q. laurina Humb. et Bonpl.

Los trabajos estatales o regionales son muy importantes para conocer acerca de la diversidad de encinos, ya que presentan listas de especies, descripciones y datos de su distribución. Entre éstos están los de McVaugh (1974), González-Villarreal (1986), Bello-González y Labat (1987), De la Cerda (1989), Vázquez (1992), RomeroRangel (1993), Valencia-A. (1995) y Espinosa-G. (2000). Trabajos más extensos son el de Martínez (1951), quien estudió los encinos de México, la obra monográfica de Trelease (1924) para los encinos de América y también la monografía de Camus (1932-1956) que trata a la familia Fagaceae para todo el mundo. Estas últimas tres obras son muy valiosas pero necesitan una revisión detallada para decidir sobre la validez, la aceptación o la sinonimia de muchos de los nombres de sus especies y así corregir errores.
Hasta el momento no existe una revisión integral y actualizada sobre la riqueza de las especies del género Quercus en México. Incluso es difícil hacer una estimación precisa de la diversidad y el endemismo del género en México. En un intento por subsanar esta deficiencia, el presente trabajo proporciona un panorama del estado del conocimiento actual sobre la diversidad de los encinos en México. Para este fin se presenta una lista preliminar de las especies del género Quercus para el país, la sinonimia respectiva para cada especie (propuestas con anterioridad y propuestas en este trabajo), así como la distribución estatal y altitudinal para cada especie (en los casos en los que esta última se conozca).

\section{Materiales y métodos}

La información presentada en la lista (apéndice 1) es el producto de datos reunidos por la autora durante más de 10 años de estudiar al género Quercus en México. En este sentido destacan las siguientes actividades.

Revisión de literatura. Se revisaron numerosas publicaciones del género Quercus sobre especies nuevas, revisiones, trabajos de complejos taxonómicos particulares, monografías, floras y listados florísticos. Estos trabajos proporcionaron las características de las especies, sinonimias, algunos datos de distribución, información de tipos y de las localidades en donde fueron colectados.

Revisión de material de herbario. Se revisaron las colecciones del género Quercus disponibles en los herbarios MEXU, FCME, BH, ENCB, y XAL, incluyendo el material tipo. También se revisaron los tipos obtenidos en préstamo de los herbarios US, C, K, F, GH, BR, NA, MO, CR, o en su defecto, fotografías de los mismos que fueron proporcionados por los herbarios P, M y K. Asimismo se revisaron las fotografías de los tipos disponibles en la página web W3TROPICOS del Missouri Botanical Garden. De esta forma se tuvo acceso a los tipos de aproximadamente $10 \%$ del total de 774 nombres que se presentan en la lista del apéndice 1. Adicionalmente, se contó con las fotografías de la publicación de Trelease (1924), que en su mayoría son tomadas de tipos.

Trabajo de campo. Se visitaron algunas localidades tipo o áreas de amplia distribución de algunas especies (principalmente en el centro de México) para conocer la variación de los taxa.

La revisión de ejemplares herborizados (o fotografías), la comparación de éstos con las descripciones (incluyendo originales) y las observaciones de campo, permitieron conocer la variación de algunas de las especies del género y corregir algunos errores detectados de determinación, de distribución estatal y de nomenclatura. De esta forma se 
obtuvo una lista inicial, la cual fue revisada con detalle y críticamente, consultando en repetidas veces la bibliografía correspondiente, las colecciones herborizadas de las especies de encinos y, en la medida de los posible, realizando trabajo de campo. Estas actividades dieron como resultado la lista preliminar de especies del género Quercus para México que se presenta aquí.

\section{Resultados}

De la lista preliminar se excluyeron 21 nombres porque se dudó de la existencia real de las especies correspondientes, ya que probablemente sólo se trate de nombres específicos dados a material que puede tratarse de: (a) un individuo originado mediante un proceso de hibridación esporádica y por lo tanto con morfología atípica, (b) alguna variación morfológica específica de alguna especie, lo cual es frecuente en renuevos formados de tocones, o (c) una especie extinta (cuadro 1).

Diversidad. Una vez excluidos dichos nombres, se encontró que en México se presentan 161 especies del género Quercus (apéndice 1). De acuerdo con los datos más recientes sobre el número estimado de especies de encinos para todo el mundo calculado entre 400 y 500 (Nixon et al.,
1997; Manos et al., 1999), en nuestro país estarían representadas entre 32.2 y $40.2 \%$ de las especies del género. Esto implica que la diversidad específica para Quercus en México equivale a casi una tercera parte respecto a la mundial.

Asimismo, teniendo en cuenta el número de especies estimadas para el continente americano (alrededor de 234), en México se encuentra $68.8 \%$ de las especies de encinos de este continente, superando así a la que se presenta en Estados Unidos, país para el que se citan 90 especies (Nixon et al., 1997), que corresponden a $38.4 \%$, y en Centroamérica, región para la que se estima que hay alrededor de 35 especies (K.C. Nixon, com. pers.), equivalentes a $15 \%$ de las especies americanas (cuadro 2).

De los dos subgéneros de Quercus (Cyclobalanopsis y Quercus), en México sólo está representado Quercus, con tres secciones: Quercus (encinos blancos) con 81 especies, Lobatae (encinos rojos) con 76 especies, y Protobalanus (encinos intermedios o de copa dorada) con cuatro de las cinco especies que en total lo conforman.

Endemismo y afinidades. Del total de especies encontradas para México, 20 también se distribuyen en Centroamérica (10 pertenecen a la sección Quercus y 10 a Lobatae). De ellas, $Q$. oocarpa Liebm., Q. pilarius Trel., Q. corrugata

Cuadro 1. Nombres de especies excluidas por su dudosa validez y datos de la publicación original.

\begin{tabular}{|c|c|}
\hline Nombre específico excluido & Referencia original \\
\hline Q. acutangula Trel. & Repert.Spec.Nov.Regni Veg. 33:318. 1934. \\
\hline Q. alamarensis C.H.Müll. & J.Arnold Arbor. 17:174. 1936. \\
\hline Q. charcasana Trel. ex A.Camus & Chênes Texte 3:216-269. 1952. \\
\hline Q. chartacea Trel. & Mem.Natl.Acad.Sci. 20:61. 1924. \\
\hline Q. floccosa Liebm. & Overs.Dansk.Vidensk.Selsk. 178. 1854. \\
\hline Q. ghiesbreghtii M.Martens et Galeotti & Bull.Acad.Roy.Sci.Bruxelles 10(2):212. 1843. \\
\hline Q. innuncupata Trel. & Mem.Natl.Acad.Sci. 20:77. 1924. \\
\hline \multirow[t]{2}{*}{ Q. juergensenii Liebm. } & Overs.Dansk.Vidensk.Selsk. 188. 1854. = Q. jurgensenii \\
\hline & A.DC. en A.D. de Candolle. Prodr. 16(2):78. 1864. \\
\hline Q. knoblochii C.H.Müll. & Am.Midl.Nat. 27:475. 1942. \\
\hline Q. manzanillana Trel. & Mem.Natl.Acad.Sci. 20:80. 1924. \\
\hline Q. microlepis Trel. et C.H.Müll. & Bull.Torrey Bot.Club 63:150. 1936. \\
\hline Q. monserratensis C.H.Müll. & Misc.Publ.U.S.Dept.Agric. 477:71. 1942. \\
\hline Q. obtusanthera Trel. & Repert.Spec.Nov.Regni Veg. 33:318. 1934. \\
\hline Q. ovandensis Matuda & Am.Midl.Nat. 43:198. 1950. \\
\hline Q. porphyrogenita Trel. & Mem.Natl.Acad.Sci. 20:51. 1924. \\
\hline Q. prinopsis Trel. & Mem.Natl.Acad.Sci. 20:61. 1924. \\
\hline Q. rekonis Trel. & Mem.Natl.Acad.Sci. 20:60. 1924. \\
\hline Q. sanchez-colini Martínez & Anales Inst.Biol.Univ.Nac.Autón.México 24:269. 1954. \\
\hline Q. schultzei Trel. & Repert.Spec.Nov.Regni Veg. 33:316. 1934. \\
\hline \multirow[t]{2}{*}{ Q. supranitida C.H.Müll. } & Am.Midl.Nat. 27:479. 1942. = Q. revoluta \\
\hline & f. acuta C.H.Müll. J.Arnold Arbor. 17:166. 1936. \\
\hline Q. vallicola Trel. & Mem.Natl.Acad.Sci. 20:81. 1924. \\
\hline
\end{tabular}


Cuadro 2. Porcentaje de riqueza de especies de encinos de México respecto a la riqueza de otros sitios de América.

\begin{tabular}{lcc}
\hline Región & Número de especies & Porcentaje \\
\hline México & 161 & 68.8 \\
Estados Unidos de América & 90 & 38.4 \\
Centroamérica & 35 & 15.0 \\
Total en América (restando & 234 & 100 \\
las 52 compartidas) & & \\
\hline
\end{tabular}

Hook et Arn., Q. oleoides Schltdl. et Cham. y Q. sapotiifolia Liebm. llegan hasta el sur de Nicaragua, mientras que el resto queda comprendido en el área definida como Megaméxico 2 por Rzedowski (1991a). Otras 33 sobrepasan la frontera norte ( 25 de la sección Quercus, 5 Lobatae y 3 Protobalanus) y solamente $Q$. polymorpha Schltdl. et Cham. se encuentra desde Estados Unidos hasta Centroamérica, atravesando el país por el centro y el este. Esto implica que existe una mayor afinidad con Estados Unidos que con Centroamérica, lo cual era de esperarse cuando se considera el origen boreal del género Quercus (Jones, 1986).

Existen 109 especies endémicas de encinos mexicanos que equivalen a $67.7 \%$ del total de especies del género en México. Este endemismo contribuye con $2.2 \%$ del total aportado por los elementos leñosos que, según Rzedowski (1991b), están representados por 4,900 especies de distribución restringida a México.

De las 109 especies que se presentan de manera natural sólo en México, 47 pertenecen a la sección Quercus, 61 a Lobatae y una a Protobalanus (cuadro 3). Estas cifras destacan un mayor número de endemismos en la sección Lobatae. Considerando además que Lobatae es una sección endémica del continente americano (Nixon, 1993b), se puede decir que México es un centro de diversidad para dicha sección.

Cuadro 3. Riqueza de especies en México, comparación con otras regiones del continenete de América y su endemismo.

\begin{tabular}{lcccc}
\hline Secciones & $\begin{array}{c}\text { Totales en } \\
\text { México }\end{array}$ & $\begin{array}{c}\text { Distribución } \\
\text { hasta } \\
\text { Centroamérica }\end{array}$ & $\begin{array}{c}\text { Distribución } \\
\text { hasta } \\
\text { Estados } \\
\text { Unidos }\end{array}$ & Endémicas \\
\hline Totales & 161 & $20^{*}$ & $33^{*}$ & 109 \\
Quercus & 81 & $10^{*}$ & $25^{*}$ & 47 \\
Lobatae & 76 & 10 & 5 & 61 \\
Protobalanus & 4 & 0 & 3 & 1 \\
\hline
\end{tabular}

* Q. polymorpha se distribuye en Centroamérica y Estados Unidos. Considerado para el conteo.
Diversidad a nivel estatal. El análisis de la riqueza estatal de especies de Quercus indica que el estado con mayor riqueza es Oaxaca con 48 especies descritas, seguido de Nuevo León con 47. A éstos le siguen Jalisco con 45, Chihuahua con 40 y Veracruz con 38 . En contraste, los que tienen menos diversidad de encinos son los estados de la Península de Yucatán: Campeche, Yucatán y Tabasco con sólo Q. oleoides. En Quintana Roo no se desarrolla ninguna especie de encino.

Dos de los estados más diversos en encinos, Oaxaca y Jalisco, podrían deber su alta riqueza específica a la compleja fisiografía determinada por la confluencia de cadenas montañosas. En Oaxaca convergen contingentes florísticos característicos de la Sierra Madre del Sur, la Sierra de Chiapas y el Eje Volcánico Transversal, lo que incrementa el número de especies en el género para tal estado. Lo mismo sucede con Jalisco, en donde convergen las floras características de la Sierra Madre Occidental, la Sierra Madre del Sur y la porción occidental del Eje Volcánico Transversal. Un caso especial es el que presenta Nuevo León con 47 especies; al respecto es necesario mencionar que Muller (1936, 1942b), en sus trabajos para las montañas del noreste de México y para el norte de México, mostró una tendencia a describir muchas especies nuevas de encinos, quizá en exceso, la mayoría de las cuales fueron colectadas en Nuevo León. Desde ese entonces no se ha realizado trabajo sistemático relativo al género Quercus en dicho estado. Por ello, es posible que futuros estudios para esa región modifiquen el estatus de algunas especies y, consecuentemente, el número de especies en la entidad.

La distribución de las especies de Quercus a nivel estatal mostró los siguientes patrones:

(1) Especies de distribución amplia. Comprende a las que se localizan en más de 15 estados y sólo están representadas por $Q$. candicans Née, $Q$. castanea Née, $Q$. crassifolia Humb. et Bonpl., $Q$. laeta Liebm., $Q$. microphylla Née, $Q$. obtusata Humb. et Bonpl., y $Q$. rugosa Née. Esta última es la que mayor distribución presenta en el territorio nacional. (2) Especies de distribución amplia media. Son 16 especies que se presentan entre 10 y 15 estados de la República Mexicana. Aquí se incluye a $Q$. chihuahuensis Trel., $Q$. deserticola Trel., $Q$. glaucoides M.Martens et Galeotti, $Q$. greggii (A.DC) Trel., Q. grisea Liebm., Q. laurina Humb. et Bonpl., $Q$. magnolifolia Née y $Q$. polymorpha, entre otras. Esta última, aunque se distribuye desde Norteamérica hasta Centroamérica, en México sólo se localiza en los estados que tienen influencia directa de la humedad proveniente del Golfo de México.

(3) Especies de distribución media. En esta categoría está la mayoría de las especies (74), que son las que se encuentran entre tres y nueve estados. Ejemplos de ellas son $Q$. arizonica Sarg., Q. corrugata Hook et Arn., Q. germana Cham. et Schltdl., Q. glaucescens Humb. et Bonpl., Q. liebmanii Oerst., $Q$. martinezii C.H.Müll., $Q$. splendens Née, 
Q. acherdophylla Trel., $Q$. aristata Hook et Arn., Q. emori Torr. y $Q$. pinnativenulosa C.H.Müll., entre otras.

(4) Especies con distribución en sólo dos estados. Corresponden a 25 especies ejemplificadas por $Q$. rubramenta Trel., $Q$. hirtiifolia, $Q$. canbyi Trel. y $Q$. benthamii A.DC.

(5) Especies con distribución en sólo un estado. Son 42 especies cuya distribución está restringida a un estado. Son ejemplos $Q$. macdougallii M.Martínez, $Q$. monterreyensis Trel. et C.H.Müll., $Q$. cualensis L.M.González, $Q$. hintoniorium Nixon et C.H.Müll. y Q. cedrosensis C.H.Müll. Entre éstas están incluidas 15 especies que además se distribuyen en Estados Unidos o en Centroamérica, siendo únicamente 27 las que se encuentran en un solo estado en la República Mexicana (cuadro 4).

Destaca el endemismo de tres especies para el estado de

Cuadro 4. Números de especies endémicas de Quercus por estado.

\begin{tabular}{lcccc}
\hline Estados & Quercus & Lobatae & Protobalanus & Totales \\
\hline Baja California & & 1 & 1 & 2 \\
Baja California Sur & 1 & 1 & 2 \\
Coahuila & 1 & 1 & 2 \\
Chiapas & & 2 & 2 \\
Chihuahua & 1 & & 1 \\
Durango & 1 & & 1 \\
Hidalgo & & 1 & 1 \\
Jalisco & & 2 & 2 \\
Nuevo León & 4 & 6 & 10 \\
Oaxaca & 1 & 2 & 3 \\
Veracruz & 1 & & 1 \\
Totales & 10 & 16 & 27 \\
\hline
\end{tabular}

Oaxaca, con una riqueza específica de 48 , así como el endemismo de la península de Baja California que es de cuatro especies (dos para Baja California y dos para Baja California Sur), con una riqueza de tan sólo 16 especies del género Quercus. En esta región seguramente el factor peninsular está jugando un papel muy importante. En el caso de Nuevo León, que es la entidad que presenta mayor número de endemismos (diez), así como una alta riqueza específica para el género, la información se debe tomar con precaución, ya que como se mencionó antes, estas cifras pueden deberse a un efecto de submuestreo y a un insuficiente trabajo taxonómico sobre género en la región.

La mayoría de las especies de Quercus presentan un endemismo regional, ya que se distribuyen de forma más o menos continua a lo largo de varios estados de una misma región. Por ejemplo, Q. martinezii se desarrolla en Jalisco,
Michoacán, Guerrero y Oaxaca, lo que muestra que fisiográficamente es exclusivo de la Sierra Madre del Sur. Quercus pinnativenulosa y $Q$. hirtiifolia sólo crecen en la Sierra Madre Oriental. Otras especies muestran distribuciones disyuntas, como por ejemplo $Q$. conzattii Trel., que crece en las montañas del noroccidente de México y en el norte de Oaxaca, y $Q$. oocarpa Liebm. qué sólo ha sido recolectada en Centroamérica y en los bosques mesófilos de montaña del occidente de México, en los estados de Guerrero, Jalisco y Nayarit.

Existen algunas especies que tienen áreas de distribución relativamente amplias, como por ejemplo $Q$. insignis M.Martens et Galeotti, $Q$. skinerii Benth. y $Q$. corrugata, entre otras; sin embargo, sus hábitats son muy restringidos y susceptibles a disturbios por la tala para diferentes actividades. Estas especies se desarrollan principalmente en el bosque mesófilo de montaña y en encinares húmedos. El tamaño de sus bellotas puede alcanzar hasta $5 \mathrm{~cm}$ de largo y esto hace que los árboles sólo las produzcan cada dos o tres años. Estas características ponen a tales especies en riesgo de desaparecer. Desafortunadamente, los datos de tipo fenológico se desconocen para la mayoría de las especies de Quercus, lo cual no sólo afecta a las especies en sí, que tienen importancia económica potencial o real, sino porque a su vez no se debe olvidar que son hábitat y fuente de alimento para roedores, aves, reptiles, insectos arácnidos y epífitas como orquídeas, bromelias, helechos y briofitas, entre los grupos más comunes. En la mayoría de los casos este tipo de relación es específica, por lo que al desaparecer el árbol, también se ve amenazada la permanencia de otros organismos.

Distribución altitudinal. De manera general los encinos se encuentran en México desde el nivel del mar hasta 3,500 m snm. Las especies de encinos blancos se distribuyen entre 0 y $3,500 \mathrm{~m}$ snm, y los intervalos por especie oscilan entre 150 y 2,000 m. Entre las especies de encinos blancos que alcanzan mayor altitud están Quercus repanda Humb. et Bonpl., $Q$. frutex Trel., $Q$. macdougalii y $Q$. glabrescens Benth., mientras que algunas especies de las que alcanzan menores altitudes son $Q$. oleoides, $Q$. magnoliifolia, $Q$. glaucescens Humb. et Bonpl. y Q. dumosa Nutt. Entre las especies con intervalos altitudinales más amplios están Quercus liebmanii, Q. magnoliifolia; Q. muehlenbergii Englem. y $Q$. obtusata. En contraste, entre los encinos blancos con intervalos altitudinales más restringidos están Quercus fusiformis Small., Q. insignis y $Q$. macdougallii.

Respecto a los encinos dorados, éstos se distribuyen entre 200 y $2,800 \mathrm{~m}$ snm, con intervalos hasta de $1,000 \mathrm{~m}$ (tal es el caso de Q. palmeri Engl.) y hasta de más de 2,000 $\mathrm{m}$ en el caso de $Q$. chrysolepis Liebm.

Los encinos rojos se distribuyen desde 150 hasta 3,100 $\mathrm{m}$ snm, con intervalos altitudinales por especie de 150 a $1,900 \mathrm{~m}$. Algunas de las especies que alcanzan mayores 
elevaciones son Quercus ocoteifolia Liebm, Q. laurina, $Q$, crassipes Humb. et Bonpl., Q. miquihuanensis Nixon et C.H.Müll. y $Q$. hintoniorum, que llegan a 3,000 m o más, mientras que los que se desarrollan a menores altitudes son $Q$. salicifolia Née, $Q$. conspersa Benth., $Q$. aristata, $Q$. elliptica Née y $Q$. macvaughii R.Spellenberg, los cuales se pueden encontrar en altitudes por debajo de $500 \mathrm{~m}$. Algunas de las especies de encinos rojos con intervalos altitudinales restringidos son Quercus duratifolia C.H.Müll., $Q$. salicifolia, $Q$. hirtiifolia y $Q$. mullerii M.Martínez, mientras que los de más amplia distribución altitudinal son $Q$. elliptica, $Q$. crassipes, $Q$. castanea, $Q$. candicans, $Q$. laurina y $Q$. acutifolia Née.

Altitudinalmente los encinos blancos presentan mayores intervalos altitudinales, tanto en general como por especie; a éstos les siguen los rojos, mientras que los de copa dorada son los más restringidos.

\section{Discusión y conclusiones}

Muchas de las especies del género Quercus en México presentan problemas taxonómicos, frecuentemente originados por el escaso conocimiento de las mismas. La forma nomenclatural de considerar a tales especies en este estudio se basó en la literatura. En este sentido es muy probable que existan diferencias entre especialistas que consideren que algunas de tales especies no deberían tener el estatus que aquí se presenta. Algunas de las entidades menos estudiadas son: Quercus aerea Trel., Q. brenesi Trel., Q. furfuraceae Liebm., $Q$. aculcingensis Trel., $Q$. ajoensis C.H.Müll., $Q$. deliquescens C.H.Müll. у $Q$. leiophylla A.DC., entre otras.

Algunas especies que se encontraban en esta situación han sido estudiadas recientemente, como es el caso de $Q$. rubramenta Trel. y $Q$. pinnativenulosa. Estas dos especies tienen distribuciones restringidas y la mayoría de los ejemplares de herbario que les corresponden estaban determinados erróneamente; actualmente ambas han sido porpuestas como especies válidas (Valencia y Jiménez-Ramírez, 1995; Valencia y Cartujano-P., 2002). Otro caso es el de Quercus ocoteifolia Liebm., el cual tradicionalmente había sido considerado como sinónimo de Q. laurina Humb. et Bonpl. Sin embargo, los trabajos de campo, herbario y gabinete muestran que se trata de una especie diferente de Q. laurina (S. Valencia, datos no publicados).

El género Quercus tradicionalmente ha sido tratado como un taxon cuyas especies, en su mayoría, presentan un alto grado de hibridación (Muller, 1952; Hardin, 1975; Grant, 1989; Bacon y Spellenberg, 1996), creándose complejos interespecíficos que reciben individualmente el nombre de singameón (Hardin, 1975 y Grant, 1989). La variación morfológica de las especies de Quercus es atribuida con frecuencia a la hibridación interespecífica. Sin embargo, a pesar de la ocurrencia de hibridación, aparentemente el origen híbrido de especies de encinos no es frecuente. De hecho, para el territorio mexicano sólo se han documentado cuatro casos de especies taxonómicas para las que se propone un origen híbrido: (1) Quercus $\times$ dysophylla Benth. cuyos progenitores son $Q$. crassifolia. y Q. crassipes (Tovar-Sánchez y Oyama, 2004); (2) Q. × basaseachisensis C.H.Müll., producto de la hibridación entre $Q$. depressipes Trel. y $Q$. rugosa Née (Spellenberg, 1995); (3) Q. $\times$ pastorensis C.H.Müll., que se origina de la hibridación de $Q$. clivicola Trel. y $Q$. glaucoides M.Martens et Galeotti (Muller, 1936); y (4) $Q . \times$ undulata Torr., cuyas especies parentales son $Q$. gambelli Nutt y $Q$. arizonica (Spellenberg, 1998).

Es importante resaltar la inclusión en la lista de seis especies que se han descrito en los últimos cinco años para México: $Q$. pachucana Zavala-Chávez, $Q$. iltisii L.M.González, $Q$. cualensis L.M.González, Q. tuitensis L.M.González, $Q$. nixoniana Valencia et Lozada y $Q$. hirtiifolia. La mayoría de ellas ya habían sido colectadas pero el material herborizado correspondiente a cada una estaba mal determinado y en los herbarios estaba intercalado entre los ejemplares de diferentes especies. Esto sólo muestra algunos ejemplos que manifiestan la necesidad de realizar estudios que permitan esclarecer problemas taxonómicos en las especies del género Quercus.

Los resultados de este trabajo mantienen a México como el país con mayor riqueza y endemismo específico para Quercus. Estos fenómenos podrían atribuirse a la combinación de varios factores. Entre los más importantes podrían estar los siguientes. En primer lugar, la fisiografía, particularmente en lo referente a las cordilleras situadas en el territorio nacional (regiones en las que se distribuye principalmente el género Quercus). Otro factor corresponde a los cambios climáticos que se han presentado desde el Pleistoceno y que han provocado desplazamientos, fragmentaciones y contactos de biotas (Jones, 1986; Rzedowski, 1991b). Un tercer factor podría ser la disponibilidad de hábitats adecuados para ser ocupados por diferentes poblaciones de encinos. Finalmente, un cuarto factor corresponería a la alta variabilidad del género (Valencia-A., 1994).

La alta riqueza específica y el elevado endemismo en el género Quercus para México ya habían sido referidos por Manos et al. (1999), quienes indican que las secciones Quercus, Lobatae y Protobalanus encontraron en México un centro de diversificación secundaria. Otros centros de diversificación secundaria son el este de Estados Unidos y Eurasia, pero el primero sólo para Lobatae y Quercus y el último sólo para Quercus (Manos et al., 1999). Las secciones Protobalanus y Lobatae sólo se distribuyen en América (Nixon, 1993b). Al respecto, Axelrod (1983) considera que la mayoría de los linajes de encinos evolucionaron localmente en latitudes medias durante el Terciario, explicando así el endemismo de las secciones del género. 
Los estados más diversos para el género Quercus en orden decreciente son Oaxaca, Nuevo León, Jalisco, Chihuahua y Veracruz. Estos datos coinciden con la propuesta de Nixon (1993a), quien establece que las zonas más ricas en México para encinos son la región del centro, sur (en donde quedan incluidos los estados de Oaxaca y Veracruz) y la región del noreste (en donde se localiza Nuevo León). Es necesario tomar en cuenta que la información de la distribución estatal de las especies de encinos, aunque útil, muestra limitantes desde una perspectiva biológica, ya que las divisiones políticas no reflejan necesariamente diferencias ambientales o históricas y pueden ser inestables a mediano plazo. Por ello, es necesario analizar la distribución específica por regiones fisiográficas, pues ello permitiría conocer más sobre la biogeografía del género y relacionar estos datos con factores ambientales e históricos. Al respecto, la autora está trabajando en la obtención de datos de distribución para las especies del género Quercus en México por región fisiográfica.

Por su parte, los datos de distribución altitudinal muestran que el intervalo en el que se desarrollan las especies de encinos en México es muy amplio, ya que abarca desde el nivel del mar hasta un altitud de 3,500 m; sin embargo, las especies se presentan más frecuentemente entre 1,000 y 3,000 m. Es necesario recordar que la sección Quercus tiene amplia tolerancia ecológica y es relativamente más diversa en áreas xéricas que la sección Lobatae (Nixon, 1993a), lo cual es congruente con lo obtenido en el presente trabajo, en donde la sección Quercus es la que tiene mayor distribución latitudinal y altitudinal.

Finalmente debe tenerse en cuenta que los datos aquí presentados son preliminares y que aún hace falta realizar mucho trabajo de campo, de herbario, de laboratorio y de gabinete que permitan resolver problemas taxonómicos en diferentes complejos de especies de encinos. Esto permitirá obtener más y mejores datos sobre diversos aspectos de las especies del género Quercus, y esto a su vez repercutirá en un mejor conocimiento para el manejo adecuado, la conservación y el aprovechamiento de uno de los taxa más importantes en los ecosistemas templados de México, el género Quercus.

\section{Agradecimientos}

Se agradece a los curadores de los herbarios MEXU, BH, ENCB, XAL, US, C, K, F, GH, BR, NA, MO, CR, P y M por facilitar la revisión de material herborizado del género Quercus, y a J. Bacon, J. Meave y a un revisor anónimo por sus valiosos comentarios y sugerencias a la versión previa de este trabajo.

\section{Literatura citada}

Axelrod D.L. 1983. Biogeography of oaks in the Arcto-Tertiary province. Annals of the Missouri Botanical Garden 70:629657.

Bacon J.R. y Spellenberg R. 1996. Hybridization in two distantly related Mexican black oaks: Quercus conzattii and Quercus eduardii (Fagaceae: Quercus: section Lobatae). Sida 17:17-41.

Bello-González M.A. y Labat J.-N. 1987. Los encinos (Quercus) del estado de Michoacán, México. Centre d’Études Mexicaines et Centroaméricaines y Secretaría de Agricultura y Recursos Hidráulicos, México, D.F.

Camus A. 1934-1955. Les Chênes. Monographie du Genre Quercus. Paul Lechevalier, París.

De la Cerda M. 1989. Encinos de Aguascalientes. Universidad Autónoma de Aguascalientes, Aguascalientes.

Espinosa-G. J. 2002. Fagaceae. En: Rzedowski J. y C. de Rzedowski G. (Eds.) Flora Fanerogámica del Valle de México, pp. 81-91, CONABIO, Instituto de Ecología, Pátzcuaro.

González R. 1993. La diversidad de los encinos mexicanos. Revista de la Sociedad Mexicana de Historia Natural 44:125142.

González-Villarreal L.M. 1986. Contribución al Conocimiento del Género Quercus (Fagaceae) en el Estado de Jalisco. Instituto de Botánica, Colección Flora de Jalisco, Universidad de Guadalajara, Guadalajara.

Govarts R. y Frodin D.G. 1998. World Checklist and Bibliography of Fagales (Betulaceae, Corylaceae, Fagaceae and Ticodendraceae). Royal Botanical Gardens, Kew.

Grant V. 1989. Especiación Vegetal. Noriega Editores, México, D.F.

Hardin J.W. 1975. Hybridization and introgression in Quercus alba. Journal of the Arnold Arboretum 56:336-363.

Jones J.H. 1986. Evolution of the Fagaceae: the implications of foliar features. Annals of the Missouri Botanical Garden 73:228-275.

Lawrence G.H. 1951. Taxonomy of Vascular Plants. MacMillan, Nueva York.

Manos P.S., Doyle J.J. y Nixon K.C. 1999. Phylogeny, biogeography, and processes of molecular differentiation in Quercus subgenus Quercus (Fagaceae). Molecular Phylogenetics and Evolution 12:333-349.

Martínez M. 1951. Los encinos de México y Centroamérica. 1. Anales del Instituto de Biología, Universidad Nacional Autónoma de México, Serie Botánica. México 22:351-368.

McVaugh R. 1974. Flora Novo-Galiciana. Contributions from the University of Michigan Herbarium 12:1-93.

Muller C.H. 1936. Studies in the oaks of the mountains of northeastern Mexico. Journal of the Arnold Arboretum 17:160-179.

Muller C.H. 1942a. The Central American species of Quercus. Miscelaneous Publication 477:1-216.

Muller C.H. 1942b. Notes on the American Flora, Chiefly Mexican. The American Midland Naturalist 27:470-490.

Muller C.H. 1952. Ecological control of hybridization in Quercus: a factor in the mechanism of evolution. Evolution 6:147-161.

Muller C.H. y McVaugh R. 1972. The oaks (Quercus) described by Née (1801), and by Humboldt \& Bonpland (1809), with comments on related species. Contributions from the University of Michigan Herbarium 9:507-522.

Nixon K.C. 1993a. The genus Quercus in Mexico. En: 
Ramammoorthy T.P., Bye R., Lot A. y Fa J. (Eds.). Biological Diversity of Mexico: Origins and Distribution, pp. 447-458, Oxford University Press, Nueva York.

Nixon K.C. 1993b. Infrageneric classification of Quercus (Fagaceae) and typification of sectional names. Annales des Sciences Forestières 50, Suppl 1:25s-34s.

Nixon K.C. y Muller C.H. 1992. The taxonomic resurrection of Quercus Laceyi Small (Fagaceae). Sida 15:57-69.

Nixon K.C. y Muller C.H. 1993. The Quercus hypoxantha complex (Fagaceae) in northeastern Mexico. Brittonia 45:146-153.

Nixon C.K., Jensen R.J., Manos P. y Muller C.H. 1997. Flora of North America, North of Mexico. Vol. 3 Magnoliophyta: Magnoliidae and Hamamelidae. Oxford University Press, Nueva York.

Romero-Rangel S. 1993. El género Quercus (Fagaceae) en el Estado de México. Tesis de Maestría, Facultad de Ciencias, Universidad Nacional Autónoma de México, México, D.F. 151 pp.

Romero S., Lira R. y Dávila P. 2000. A phenetic study of the taxonomic delimitation of Quercus acutifolia and Q. conspersa (Fagaceae). Brittonia 52:177-187.

Rzedowski J. 1991a. Diversidad y orígenes de la flora fanerogámica de México. Acta Botanica Mexicana 14:3-21.

Rzedowski J. 1991b. El endemismo en la flora fanerogámica mexicana: una apreciación analítica preliminar. Acta Botanica Mexicana 15:47-64.

Spellenberg R. 1995. On the hybrid nature of Quercus basaseachicensis (Fagaceae, sect. Quercus). Sida 16:427-434.

Spellenberg R. 1998. Quercus lesueri, un miembro meridional del complejo de $Q$. x undulata (Fagaceae, subgénero Quercus). Acta Botanica Mexicana 42:25-33.

Spellenberg R. y Bacon J.R. 1996. Taxonomy and distribution of a natural group of black oaks of Mexico (Quercus, section Lobatae, subsection Racemiflorae). Systematic Botany 21:85-99.

Tovar-Sánchez E. y Oyama K. 2004. Natural hybridization and hybrid zones between Quercus crassifolia and Q. crassipes (Fagaceae) in Mexico: morphological and molecular evidence. American Journal of Botany 91:1352-1363.

Trelease W. 1924. The American oaks. Memoirs of the National Academy of Sciences 20:1-255, 420 pl.

Valencia-A. S. 1994. Contribución a la delimitación taxonómica de tres especies del género Quercus subgénero Erythrobalanus: Q. laurina Humboldt et Bonpland, Q. affinis Sheidweiler y $Q$. ghiesbregtii Martens et Galeotti. Tesis de Maestría, Facultad de Ciencias, Universidad Nacional Autónoma de México, México, D.F., 174 pp.

Valencia-A. S. 1995. Contribución al Conocimiento del Género Quercus (Fagaceae) en el Estado de Guerrero, México. Contribuciones del Herbario de la Facultad de Ciencias No. 1, Universidad Nacional Autónoma de México, México, D.F.

Valencia-A. S. y Jiménez-Ramírez J. 1995. Redescripción de Quercus rubramenta Trel. Anales del Instituto de Biología, Universidad Nacional Autónoma de México, Serie Botánica 61:5-10.

Valencia-A. S.y Cartujano-P. S.L. 2002. Quercus pinnativenulosa (Fagaceae), un encino poco conocido de la Sierra Madre Oriental. Anales del Instituto de Biología, Universidad Nacional Autónoma de México, Serie Botánica 73:87-92.

Vázquez M.L. 1992. El género Quercus (Fagaceae) en el estado de Puebla, México. Tesis de Licenciatura, ENEP-Zaragoza, Universidad Nacional Autónoma de México, México, D.F., $246 \mathrm{pp}$.

Vázquez M.L., Valencia-A. S. y Nixon K.C. 2004. Notes on red oaks (Quercus sect. Lobatae) in eastern Mexico, with description of a new species. Quercus hirtiifolia. Brittonia 56:136142.

Zavala-Chávez F. 1995. Encinos Hidalguenses. Ediciones Universidad Autónoma de Chapingo, Chapingo, 133 pp.

Fecha de recepción: 26 de mayo de 2004

Versión corregida: 28 de octubre de 2004

Aceptado: 29 de octubre de 2004 
Apéndice 1. Lista preliminar de especies del género Quercus L. subgénero Quercus (Fagaceae) en México y su sinonimia propuesta $\left(\mathrm{CAm}=\right.$ Centroamérica; $\mathrm{EUA}=$ Estados Unidos de América). El asterisco $\left({ }^{*}\right)$ indica los sinónimos que fueron propuestos en este trabajo.

\author{
A. Sección Lobatae Loudon (encinos rojos). \\ 1. Q. acherdophylla Trel., Mem.Natl.Acad.Sci. 20:183. 1924. \\ Distribución estatal en México: Hgo., Pue. y Ver. Altitud: 2400-2500 m. \\ 2. Q. acutifolia Née, An.Ci.Nat. 3:267. 1801. \\ =Q. acutifolia f. abrupta Trel.; Q. acutifolia var. angustifolia A.DC.; Q. acutifolia var. bonplandii A.DC. y Q. sartorii \\ Botteri ex A.DC. \\ Distribución estatal en México: Gro., Jal., Méx., Mich., Mor., Nay., Oax. y Pue. Altitud: 1000-2500 m.
}

3. Q. aerea Trel., Mem.Natl.Acad.Sci. 20:135. 1924.

Distribución estatal en México: Chih. y SLP.

4. Q. affinis Scheidw., L'Horticulteur Belge 4:321, pl. 17. 1837.

= Q. affinis f. commutata Trel.; Q. affinis f. subintegra Trel.; Q. commutata Liebm.; Q. nitens M.Martens et Galeotti; Q. nitens var. subintegra A.DC.

Distribución estatal en México: Gto., Hgo., NL, Oax., Pue., Qro., SLP, Tamps. y Ver. Altitud: 1200-2600 m.

5. Q. agrifolia Neé, An.Ci.Nat. 3:271. 1801.

=Q. acroglandis Kellogg; Q. acutiglandis Sarg.; Q. agrifolia var. frutescens Engelm.; Q. agrifolia var. oxyadenia (Torr.) J.T.Howell; Q. oxyadenia Torr. ex Sitgr.; Q. pricei Sudw.

Distribución estatal en México: BC (península). También se distribuye en EUA. Altitud: ca. 1400 m.

6. Q. albocincta Trel., Mem.Natl.Acad.Sci. 20:193. 1924.

Distribución estatal en México: Chih., Dur., Sin. y Son. Altitud: 1100-1800 m.

7. Q. aristata Hook. et Arn. Bot.Beech.Voy. 444. 1841.

=Q. productipes Trel.

Distribución estatal en México: Ags., Jal., Nay. y Sin. Altitud: 600-1750 m.

8. Q. benthamii A.DC. in A.P. de Candolle, Prodr. 16(2):29. 1864.

=Q. gemmata Trel.; Q. undulata Benth.

Distribución estatal en México: Chis. y Oax. También se distribuye en CAm (Guatemala). Altitud: $1500-3000$ m.

9. Q. canbyi Trel., Mem.Natl.Acad.Sci. 20:188. pl. 379 y 380. 1924.

$={ }^{*}$ Q. alamarensis C.H.Müll.; Q. canbyi f. attenuata C.H.Müll.; Q. canbyi f. berlandieri Trel.; Q. canbyi f. concolor Trel. et C.H.Müll.; Q. canbyi f. karwinskii Trel.; Q. canbyi f. pedunculata C.H.Müll.; Q. canbyi f. setacea C.H.Müll.; Q. canbyi f. subovatifolia C.H.Müll.; Q. canbyi f. typica A.Camus; Q. karwinskii Trel.

Distribución estatal en México: NL y Tamps. Altitud: 650-2000 m.

10. Q. candicans Née, An.Ci.Nat. 3:277. 1801.

= Q. acuminata M.Martens et Galeotti; Q. alamo Benth.; Q. calophylla f. acuminata Trel.; Q. calophylla f. alamo Trel.; Q. calophylla f. flavida Trel.; Q. calophylla f. schiedeana Trel.; Q. calophylla f. willdenovii Trel.; Q. calophylla Schltdl. et Cham.; Q. callophylla f. intermedia (M.Martens et Galeotti) Seem ex Trel.; Q. callophylla f. michoacana. Trel.; Q. callophylla var tipica Camus; Q. candicans f. incurva Trel.; Q. chimaltenangensis f. gemmata C.H.Müll.; Q. flavida Liebm.; Q. intermedia M.Martens et Galeotti; Q. umbrosa Endl.; Q. candicans f. michoacana Trel.; Q. candicans var. alligata Trel.

Distribución estatal en México: Chih., Chis., DF, Dgo., Gro., Hgo., Jal., Méx., Mich., Mor., Nay., Oax., Pue., Sin. y Ver. También crece en CAm (Guatemala). Altitud: 1400-2600 m.

11. Q. castanea Née, An.Ci.Nat. 3:276. 1801.

= Q. alamosensis Trel.; Q. axilaris Trel.; Q. castanea var. elliptica Trel.; Q. circummontana Trel.; Q. crassivenosa Trel. Q. impresa Trel.; Q. lanigera M.Martens et Galeotti; Q. lanigera f. siderosyloides Trel.; Q. mucronata Willd.; Q. pulchella 
Humb. et Bonpl.; Q. rossii Trel.; Q. rossii f. arsenei Trel.; Q. seleri Trel.; Q. serrulata Trel.; Q. simillima Trel.; Q. spathulistipula Trel.; Q. subcrispata Trel.; Q. tepoxuchilensis Trel.; Q. tepoxuchilensis f. perplexans Trel.; Q. tristis Liebm.; Q. verrucosirana Trel.

Distribución estatal en México: Chis., Col., DF, Dgo., Gto., Gro., Hgo., Jal., Méx., Mich., Mor., Nay., Oax., Pue., Sin., SLP, Son. y Ver. También se distribuye en CAm (Guatemala y El Salvador). Altitud: 1180-2600 m.

12. Q. coahuilensis Nixon et C.H.Müll., Brittonia 45:150. 1993.

Distribución estatal en México: Coah. y Chih. Altitud: 2000-3550 m.

13. Q. coccolobifolia Trel., Mem.Natl.Acad.Sci. 20:136. 1924.

Q. jonesii Trel.; Q. endlichiana Trel.

Distribución estatal en México: Chih., Jal., Nay., SLP y Son. Altitud: 1550-2300 m.

14. Q. conspersa Benth., PI.Hartw. 91. 1842.

= Q. acutifolia var. conspersa A.DC.; Q. acutifolia var. microcarpa A.DC.; *Q. candolleana Trel.; Q. conspersa f. caudata Trel.; Q. conspersa f. ovatifolia Trel.; Q. nitida M.Martens et Galeotti; Q. grahami var. coyulana Trel.; Q. grahami var. nelsoni Trel.; Q. tonaguiae Trel.; Q. uruapanensis Trel.; *Q. vexans Trel.

Distribución estatal en México: Chis., Gro., Jal., Méx., Mich., NL, Oax. y Ver. Altitud: 540-2380 m.

15. Q. conzattii Trel., Proc.Amer.Phil.Soc. 60:33. 1921.

= Q. urbanii f. parvifolia M.Martínez.

Distribución estatal en México: Dgo., Jal., Nay., Oax. y Zac. Altitud: 1700-2600 m.

16. Q. cortesii Liebm., Overs.Dansk.Vidensk.Selsk. 175. 1854.

$={ }^{*} Q$. brenessi Trel.

Distribución estatal en México: Chis., Oax. y Ver. Altitud: 1800 m.

17. Q. crassifolia Humb. et Bonpl., Pl.Aequinoct. 2(43):49, pl. 91. 1801.

= Q. brachystachys Benth.; Q. chicamolensis Trel.; Q. errans Trel.; Q. felipensis Trel.; Q. miguelitensis Trel.; Q. mollis M.Martens et Galeotti; Q. moreliana Trel.; Q. orbiculata Trel.; Q. spinulosa M.Martens et Galeotti; Q. splendens var. pallidior A.DC.; Q. stipularis Humb. et Bonpl.

Distribución estatal en México: Chih., Chis., DF, Dgo., Gto., Gro., Hgo., Jal., Méx., Mich., Oax., Pue., Qro., SLP, Tlax., Ver. y Zac. También llega hasta CAm (Guatemala). Altitud: 1300-2900 m.

18. Q. crassipes Humb. et Bonpl., PI.Aequinoct. 2(10):37-38 pl. 83. 1809.

Q. castanea var. glabrata (Liebm. ex Seem. ) Trel.; Q. colimae f. zauzillo Trel.; Q. colimae Trel.; Q. confertifolia Humb. et Bonpl.; Q. crassipes var. angustifolia Humb. et Bonpl.; Q. cuajimalpana Trel.; Q. imbricariaefolia Trel.; Q. malifolia Trel.; Q. mexicana Benth.; Q. mexicana f. confertifolia (Bonpl.) Trel.; Q. mexicana sensu Trel.; Q. mexicana var. glabrata Liebm. ex Seem.; Q. mexicana f. angustifolia (Bonpl.) Trel.; Q. obovalifolia Trel.

Distribución estatal en México: Col., DF, Gto., Hgo., Jal., Méx., Mich., Mor., Oax., Pue., Qro. y Tlax. Altitud: 2000$2600 \mathrm{~m}$.

19. Q. crispifolia Trel., Mem.Natl.Acad.Sci. 20:147. 1924.

= Q. amphioxys Trel.; Q. incrassata Trel.

Distribución estatal en México: Chis., Gro. y Oax.; también llega hasta CAm (Guatemala); Altitud: 750-1500 m.

20. Q. crispipilis Trel., Mem.Natl.Acad.Sci. 20:184. 1924.

= Q. brachystachys f. caerulea Trel.; Q. cerífera Trel.; Q. cinnamomea Trel.; Q. skutchii Trel.

Distribución estatal en México: Chis. También se distribuye en CAm (Guatemala). Altitud: 1800-2420 m.

21. Q. cualensis L.M.González, Brittonia 55(1):49-60. 2003.

Distribución estatal en México: Jal. Altitud: 1800-2300 m.

22. Q. cupreata Trel. et C.H.Müll., Bull.Torrey Bot.Club 63:152. 1936.

= Q. cupreata f. brachystachys C.H.Müll.; Q. cupreata f. serrata Trel.

Distribución estatal en México: NL. 
23. Q. depressa Humb. et Bonpl., Pl.Aequinoct. 2(10):50, pl. 92:1809.

=Q. subavenia Trel. Q. laurina M.Martens et Galeotti.

Distribución estatal en México: Hgo., Oax., Pue. y Ver. Altitud: 1800-2600 m.

24. Q. devia Goldman, Contr.U.S.Natl.Herb. 16:322. 1916.

Distribución estatal en México: BCS. Altitud: 2600 m.

25. Q. duratifolia C.H.Müll., Misc.Publ.U.S.Dept.Agric. 477:50. 1942.

Distribución estatal en México: Chis.

26. Q. durifolia von Seemen ex Loes., Bot.Jahrb.Syst. 29:95. 1900.

Distribución estatal en México: Chih., Dgo., Sin. y Son. Altitud: 1700-2300 m.

27. Q. $\times$ dysophylla Benth., PI. Hartweg. 55. 1840. (Q. crassifolia Humb. et Bonpl. $\times$ Q. crassipes Humb. et Bonpl.)

= Q. esperanzae Trel.; Q. fournieri Trel.; Q. hahnii Trel.

Distribución estatal en México: DF, Gto., Hgo., Méx., Mich., Tlax. y Ver. Altitud: 2490-2850 m.

28. Q. eduardii Trel., Contr.U.S.Nat.Herb. 23:189. 1922.

= Q. eduardii f. cespitifera Trel.; Q. nitidissima Trel.; Q. oligodonta Seemen ex Loes.

Distribución estatal en México: Ags., Chih., Dgo., Gto., Hgo., Jal., Mich., Nay., Qro., SLP, Tamps., Ver. y Zac. Altitud: 1500-2650 m.

29. Q. elliptica Née., An.Ci.Nat. 3:278. 1801.

$={ }^{*} Q$. atrescentirhachis Trel.; Q. botryocarpa Trel.; *Q. coccinata Trel.; Q. comayaguana Trel.; Q. chiquihuitillonis Trel.; Q. exaristata Trel.; Q. guayabalana Trel. ex Standl.; Q. hondurensis Trel. Q. langlassei Trel.; Q. linguifolia Liebm.; Q. nectandraefolia Liebm.; Q. oajacana Liebm.; Q. oaxacana Trel.; Q. peradifolia Warb.; *Q. porriginosa Trel.; Q. pubinervis M.Martens et Galeotti; Q. yoroensis Trel.; Q. yoroensis var. aguanana Trel.; Q. lanceolata M.Martens et Galeotti ex A.DC.

Distribución estatal en México: Chis., Gro., Jal., Méx., Mich., Nay., Oax., Sin. y Ver. También se distribuye en CAm (Honduras, El Salvador y Guatemala). Altitud: 470-2460 m.

30. Q. emoryi Torr. in W.H.Emory, Not.Milit.Reconn. 151:pl. 9. 1848.

= Q. balsequillana Trel.; Q. duraznillo Trel.; Q. duraznillo f. bullata Trel.; Q. duraznillo f. cochutensis Trel.; Q. duraznillo f. pinetorum Trel.; $Q$. hastata Liebm.

Distribución estatal en México: Chih., Coah., Dur., NL, Son. y Tamps. También se distribuye en EUA. Altitud: 1000$2200 \mathrm{~m}$.

31. Q. flocculenta C.H.Müll., J.Arn.Arbor. 17:172. 1936.

= Q. flocculenta f. incisa C.H.Müll.; Q. flocculenta f. oblongifolia C.H.Müll.; Q. flocculenta var. typica A.Camus.

Distribución estatal en México: NL. Altitud: 2300-2800 m.

32. Q. fulva Liebm., Overs.Dansk.Vidensk.Selsk. 183. 1854.

$=$ Q. rosei Trel.

Distribución estatal en México: Chih., Dgo., Jal., Nay. y Sin. Altitud: 1900-2900 m.

33. Q. furfuraceae Liebm., Overs.Dansk.Vidensk.Selsk. 189. 1854.

$=Q$. acutifolia var. furfuraceae (Liebm.) Oerst.

Distribución estatal en México: Mich. y Pue. Altitud: 2300-2600 m.

34. Q. galeanensis C.H.Müll., J.Arn.Arbor. 17:173. 1936.

Distribución estatal en México: NL. Altitud: 1200 m.

35. Q. gentry C.H.Müll., Am.Midl.Nat. 27:474. 1942.

Distribución estatal en México: Chih., Jal., Mich., Nay., Sin. y Zac. Altitud: 1250-2400 m.

36. Q. graciliramis C.H.Müll., J.Arn.Arbor. 17:177 (1936).

Distribución estatal en México: NL. Altitud: 1900-2100 m. 
37. Q. grahami Benth., PI.Hartw. 57. 1840.

=Q. grahami f. brevipes Trel.

Distribución estatal en México: Oax. Altitud: 1000-2000 m.

38. Q. gravesii Sudworth, Check List For. USDA.Misc.Circ. 92:86. 1927.

= Q. chesosensis (Sarg.) C.H.Müll.; Q. coccinea var. microcarpa Torr.; Q. shumardii Buckley var. microcarpa (Torr.) Shinners; Q. stellapila (Sarg.) H.B.Parks ex Cory; Q. texana var. chesosensis Sarg.; Q. texana var. stellapila Sarg.

Distribución estatal en México: Coah. También se distribuye en EUA. Altitud: $1200 \mathrm{~m}$.

39. Q. hintonii E.F.Warb., Bull.Misc.Inform.Kew 91. 1939.

= Q. apiophylla E.F.Warb.; Q. ochroesthes E.F.Warb.; Q. sagata E.F.Warb.

Distribución estatal en México: Méx. y Mich. Altitud: 1300-1950 m.

40. Q. hintoniorium Nixon et C.H.Müll., Brittonia 45:147. 1993.

Distribución estatal en México: Coah. Altitud: 2600-3300 m.

41. Q. hirtifolia Vázquez-Villagrán, Valencia y Nixon. Brittonia 56(2):136-142. 2004.

Distribución estatal en México: Hgo. y Pue. Altitud: 2000-2400 m.

42. Q. hypoleucoides A.Camus, Bull.Mus.Natl.Hist.Nat., Ser. 2, 4:124. 1932.

= Q. hypoleuca Engelm.; Q. confertifolia Torr.

Distribución estatal en México: Chih., Coah., Dgo. y Son. También se desarrolla en EUA. Altitud: $1100-2700$ m.

43. Q. hypoxantha Trel., Mem.Natl.Acad.Sci. 20:170. 1924.

$=Q$. errans f. graciliramis C.H.Müll.

Distribución estatal en México: Coah., NL y SLP. Altitud: 2100-2925 m.

44. Q. iltisii L.M.González, Brittonia 55(1):49-60. 2003.

Distribución estatal en México: Col. y Jal. Altitud: 330-1550 m.

45. Q. laurina Humb. et Bonpl., Pl.Aequinoct. 2(10):32 pl. 80. 1809.

= Q. barbinervis Benth.; Q. bourgaei Trel.; Q. bourgaei var. ilicifolia Trel.; Q. caeruleocarpa Trel.; ${ }^{*} Q$. castanea var. tridens A.DC.; Q. chrysophylla Humb. et Bonpl.; Q. lanceolata Humb. et Bonpl.; *Q. laurina var. barbinervis (Benth.) Wenz.; ${ }^{*}$ Q. laurina var. major (A.DC.) Wenz.; Q. major (A.DC) Trel.; ${ }^{*} Q$. malinaltepecana Trel.; ${ }^{*} Q$. nitens var. major A.DC.; Q. orizabae Liebm.; Q. roseovenulosa Trel.; Q. salicifolia var. tlapuxahuensis (A.DC.) Wenz.; Q. tlapuxahuensis A.DC.; Q. tlapuxahuensis var. obconica Trel.; Q. treleasana A.Camus; Q. tridens Humb. et Bonpl.

Distribución estatal en México: DF, Gro., Gto., Hgo., Jal., Méx., Mich., Mor., Oax., Pue., Qro., Tlax. y Ver. Altitud: 1600-3000 m.

46. Q. macvaughii R.Spellenberg, Am.J.Bot. 79(10):1200-1206. 1992.

Distribución estatal en México: Chih., Dgo. y Son.; Altitud: 150-3100 m.

47. Q. mexicana Humb. et Bonpl., Pl.Aequinoct. 2(10):35 pl. 82. 1809.

=Q. castanea var. integra Oerst.; Q. castanea var. mexicana (Bonpl.) A.DC.; Q. malifolia Trel. Q. mexicana f. lanulosa Trel.; Q. mexicana f. perfertilis Trel.; Q. pablillensis C.H.Müll.; Q. rugulosa M.Martens et Galeotti.

Distribución estatal en México: Coah., DF, Hgo., Méx., NL, Pue., Qro., SLP, Tamps., Tlax. y Ver. Altitud: 2230-3050 m.

48. Q. miquihuanensis Nixon et C.H.Müll., Brittonia 45:152. 1993.

Distribución estatal en México: Tamps. y NL. Altitud: 2500-2900 m.

49. Q. mulleri Martínez An.Inst.Biol.Univ.Nac.Aut.México 24:51. 1953.

Distribución estatal en México: Oax. Altitud: 1000-1800 m.

50. Q. nixoniana Valencia y Lozada, Novon, 13:261-264. 2003.

Distribución estatal en México: Gro., Jal. y Oax.; Altitud: 1300-2300 m.

51. Q. ocoteifolia Liebm., Overs.Dansk.Vidensk.Selsk. 176. 1854. 
$={ }^{*} Q$. acatenangensis Trel.; ${ }^{*} Q$. acutifolia var. longifolia A.DC.; ${ }^{*} Q$. ambivenulosa Trel.; ${ }^{*} Q$. donell-smithii Trel. (pro parte); ${ }^{*} Q$. esesmilensis Tucker et C.H.Müll.; ${ }^{*} Q$. laurina var. beta podocarpa Wenz.; ${ }^{*} Q$. laurina var. ocoteaefolia Wenz.; ${ }^{*} Q$. Iongifolia Liebm.; ${ }^{*} Q$. nitens var. ocoteaefolia (Liebm.) A.DC.; ${ }^{*} Q$. nitens var. podocarpa A.DC.; ${ }^{*} Q$. ocot[sic]aefolia Liebm.-Oersted; ${ }^{*} Q$. ocoteaefolia f. confusa Trel.; ${ }^{*} Q$. ocoteaefolia f. pododcarpa Trel.; ${ }^{*} Q$. trinitatis Trel.; *Q. xalapensis var. longifolia Wenz.; ${ }^{*} Q$. zempoaltepecana Trel.

Distribución estatal en México: Chis., Hgo., Oax. y Ver. También se distribuye en CAm (El Salvador y Guatemala). Altitud: $1750-3000 \mathrm{~m}$.

52. Q. pachucana Zavala-Chávez, J.Int.Oaks Soc. 10:30-35. 2000.

Distribución estatal en México: Hgo. Altitud: 2800-2900 m.

53. Q. paxtalensis C.H.Müll., Misc.Publ.U.S.Dept.Agric. 477:75. 1942.

Distribución estatal en México: Chis.

54. Q. peninsularis Trel., Mem.Natl.Acad.Sci. 20:124. 1924.

Distribución estatal en México: $B C$.

55. Q. planipocula Trel., Mem.Natl.Acad.Sci. 20:136. 1924.

$=Q$. planipocula f. subsessilifolia Trel.

Distribución estatal en México: Gro., Jal., Mich., Nay. y Sin. Altitud: 680-1960 m.

56. Q. pinnativenulosa C.H.Müll., J.Arn.Arb. 17:171. 1936.

Distribución estatal en México: NL, Qro., SLP, Tamps. y Ver. Altitud: 800-1600 m.

57. Q. prainiana Trel., Mem.Natl.Acad.Sci. 20:138. 1924.

$=Q$. aequivenulosa Trel.; Q. coffeicolor Trel.

Distribución estatal en México: Nay., Jal. y Sin. Altitud: 900-1900 m.

58. Q. radiata Trel., Proc.Amer.Phil.Soc. 60: 33. 1921.

Distribución estatal en México: Dgo., Jal. y Zac. Altitud: 2000-2600 m.

59. Q. rhysophylla Weatherby, Proc.Amer.Acad. 45:423. 1910.

Distribución estatal en México: Hgo., NL y Ver. Altitud: 1400-1900 m.

60. Q. rubramenta Trel., Rep.Spec.Nov.Regni Veg. 33:318. 1934.

Distribución estatal en México: Gro. y Oax. Altitud: 2200-2800 m.

61. Q. runcinatifolia Trel. et C.H.Müll., Bull.Torrey Bot.Club 63:153. 1936.

= Q. runcinatifolia f. lata Trel. et C.H.Müll.; Q. runcinatifolia var. typica A.Camus;

Distribución estatal en México: NL.

62. Q. salicifolia Née, An.Ci.Nat. 3:265. 1801.

=Q. acapulcensis Trel.; Q. tahuasalana Trel.

Distribución estatal en México: Gro., Jal. y Mich. Altitud: 500-750 m.

63. Q. saltillensis Trel., Mem.Natl.Acad.Sci. 20:pl. 368. 183. 1924.

= Q. carnerosana Trel.; Q. sierramadrensis C.H.Müll.; Q. tenuiloba f. gracilis C.H.Müll.

Distribución estatal en México: Coah. y NL. Altitud: 1900-2400 m.

64. Q. sapotiifolia Liebm., Overs.Dansk.Vidensk.Selsk. 185. 1854.

= Q. amissiloba Trel.; Q. apanecana Trel.; Q. bumelioides Liebm.; Q. correpta Trel.; Q. donnellsmithii Trel. (pro parte); ${ }^{*}$ Q. elliptica Liebm. ex A.DC. Q. elliptica var. microcarpa (Liebm.) A.DC. Q. guatimalensis A.DC. (pro parte); Q. microcarpa Liebm.; Q. parviglans f. polycarpa Trel.; Q. parviglans f. tejadana Trel.; Q. parviglans Trel.; *Q. persiifolia f. microcarpa Trel.; *Q. persiifolia Liebm.; Q. persiifolia var. ahoteana Trel. ex Yunck.; Q. siguatepequeana Trel.; Q. totutlensis A. DC; Q. wesmaelii Trel.

Distribución estatal en México: Chis., Hgo., Oax. y Ver. También se localiza en CAm (Costa Rica, Guatemala, Honduras, Panamá, El Salvador). Altitud: 250-2000 m. 
65. Q. sartorii Liebm. Overs.Dansk.Vidensk.Selsk. 177. 1854.

$={ }^{*} Q$. huitamalcana Trel.; Q. serra Liebm. (no Q. serra Unger); Q. sartorii f. magna Trel.

Distribución estatal en México: Hgo., Pue., Oax., Tamps. y Ver. Altitud: 1300-2000 m.

66. Q. scytophylla Liebm. Overs.Dansk.Vidensk.Selsk. 180. 1854.

=Q. campanariensis Trel.; Q. epileuca Trel.

Distribución estatal en México: Gro., Jal., Méx., Mich., Nay., Oax. y Pue. Altitud: 900-2600 m.

67. Q. sideroxyla Humb. et Bonpl., Pl.Aequinoct. 2(10):39. 85. 1809.

= Q. incarnata Trel.; Q. omissa A.DC.; Q. sideroxyla f. aquifolia Trel.; Q. sideroxyla f. ciliifera Trel. Q. incarnata f. ampla Trel., Q. incarnata f. grosseserrata Trel., Q. incarnata f. longa Trel.

Distribución estatal en México: Ags., Chih., Coah., Dgo., Gto., Jal., Nay., NL, SLP, Son., Tamps. y Zac. Altitud: 2000$2650 \mathrm{~m}$.

68. Q. skineri Benth., Gard.Chron. 1841:16. 1841.

= Q. chiapasensis f. cuneifolia Trel.; Q. chiapasensis f. falcilobata Trel.; Q. chiapasensis f. flagellata Trel.; Q. chiapasensis f. longipes Trel.; Q. chiapasensis f. petiolata Trel.; Q. chiapasensis f. subcuneata Trel.; Q. chiapasensis Trel.; Q. grandis Liebm.; Q. grandis var. tenuipes Trel.; Q. hemipteroides C.H.Müll.; Q. salvadorensis Trel.; Q. trichodonta Trel.

Distribución estatal en México: Chis. y Oax. También se desarrolla en CAm (Guatemala, Honduras, El Salvador). Altitud: ca.1500 m.

69. Q. tarahumara R.Spellenberg, J.Bacon, D.Breedlove, Madroño 42(1):26-33. 1995.

Distribución estatal en México: Chih., Dgo., Sin. y Son. Altitud: 1100-2000 (2200) m.

70. Q. tardifolia C.H.Müll., Bull.Torrey Bot.Club 63:154. 1936.

Distribución estatal en México: Coah. También se distribuye en EUA. Altitud: ca. 2000 m.

71. Q. tenuiloba C.H.Müll., J.Arn.Arbor. 17:178. 1936.

= Q. tenuiloba f. hirsuta C.H.Müll.

Distribución estatal en México: NL. Altitud: 1400-1900 m.

72. Q. tuitensis González-Villarreal. Brittonia 55(1):42-48. 2003.

Distribución estatal en México: Jal. Altitud: 980-1400 m.

73. Q. urbanii Trel., Proc.Am.Philosoph.Soc. 60:32. pl. 2. 1921.

= Q. pennivenia Trel.

Distribución estatal en México: Dgo., Gro., Méx., Nay. y Sin. Altitud: 1400-2200 m.

74. Q. uxoris McVaugh., Contr.Univ.Mich.Herb. 9:513. 1972.

Distribución estatal en México: Col., Gro., Jal., Mich. y Oax. Altitud: 1500-2500 m.

75. Q. viminea Trel., Mem.Natl.Acad.Sci. 20: 123. pl. 222. 1924.

$=$ Q. bolanyosensis Trel.

Distribución estatal en México: Ags., Chih, Dgo., Gto., Jal., Nay., Sin. y Son. También se distribuye en EUA. Altitud: 1500-2100 m.

76. Q. xalapensis Humb. et Bonpl., Pl.Aequinoct. 2:24. 1809.

= Q. xalapensis f. jalapae Trel.; $Q$. xalapensis f. surculina Trel.

Distribución estatal en México: Hgo., SLP, Tamps. y Ver. Altitud: 1800 m.

B. Sección Quercus (encinos blancos)

77. Q. aculcingensis Trel., Contr.U.S.Natl.Herb. 23:185. 1922.

$=Q$. reticulata var. crassifolia Oerst.; Q. reticulata var. retifolia Liebm.

Distribución estatal en México: Ver. Altitud: ca. 2200 m.

78. Q. ajoensis C.H.Müll., Madroño 12:140. 1954. 
= Q. turbinella subsp. ajoensis (C.H.Müll.) Felger et C.H.Lowe.; Q. turbinella var. ajoensis (C.H.Müll.) Little.

Distribución estatal en México: BC (península). También se distribuye en EUA. Altitud: 500-1500 m.

79. Q. alpescens Trel., Mem.Natl.Acad.Sci. 20:83. pl. 122. 1924.

Distribución estatal en México: Hgo. y NL.

80. Q. arizonica Sarg., Gard.Forest 8:92. 1895.

= Q. arizonica var. wootonii Trel.; Q. endemica C.H.Müll.; Q. sacame Trel.

Distribución estatal en México: Chih., Coah., Dgo. y Son. También se localiza en EUA. Altitud: 1300-2500 m.

81. Q. × basaseachicensis C.H.Müll., Am.Midl.Nat. 19: 582. 1938. (Q. depressipes Trel. × Q. rugosa Née).

Distribución estatal en México: Chih. y Dgo. Altitud: 1980-2225 m

82. Q. brandegei Goldman, Contr.U.S.Nat.Herb. 16:321. 1916.

Distribución estatal en México: BCS.

83. Q. carmenensis C.H.Müll., Amer.Midl.Nat. 18:847. 1937.

Distribución estatal en México: Coah. También se localiza en EUA. Altitud: 2200-2500 m

84. Q. chihuahuensis Trel., Mem.Natl.Acad.Sci. 20:85. 1924.

=Q. chihuahensis f. amplifolia trel., Q. chihuahensis f. microphylloides Trel.; Q. chihuahuensis f. tenuis Trel.; Q. infralutea Trel.; Q. jaliscensis Trel.; Q. santaclarensis C.H.Müll.

Distribución estatal en México: Ags., Chih., Dgo., Jal., Nay., NL, Sin., SLP, Son. y Zac. También se localiza en EUA. Altitud: $1100-2700 \mathrm{~m}$.

85. Q. clivicola Trel. et C.H.Müll., Bull.Torrey Bot.Club 63:149. 1936.

= Q. clivicola f consanguinea C.H.Müll.; Q. clivicola f. crenifolia Trel. et C.H.Müll.; Q. clivicola f. dentata Trel. et C.H.Müll.; Q. clivicola f. elongata C.H.Müll.

Distribución estatal en México: NL y Tamps. Altitud: 900-2200 m

86. Q. convallata Trel., Mem.Natl.Acad.Sci. 20:88. 1924.

= Q. reticulata f. apus (Trel.) A.Camus; Q. reticulata f. inclusa (Trel.) A.Camus; Q. rhodophlebia f. apus Trel.; Q. rhodophlebia f. inclusa Trel.

Distribución estatal en México: Dgo., Jal., Nay. y Zac. Altitud: 2300-2500 m

87. Q. cordifolia Trel., Mem.Natl.Acad.Sci. 20:84. 1924.

Distribución estatal en México: Coah., NL, SLP y Zac.

88. Q. cornelius-mulleri Nixon et K.P.Steele, Madroño 28:210. 1981.

Distribución estatal en México: BC. También se distribuye en EUA. Altitud: 1000-1800 m.

89. Q. corrugata Hook et Arn., Icon.PI. V. pl. 403-4. 1842.

= Q. corrugata var. granulifera Trel.; Q. corrugata var. ipalensis Trel.; Q. corrugata var. microcarpa Wenz.; Q. cyclobalanoides Trel.; Q. pilgeriana Seem. Q. reevesii Trel.

Distribución estatal en México: Chis., Gro., Oax., Pue. y Ver. También se distribuye en CAm (Guatemala, Honduras, El Salvador, C. Rica). Altitud: 850-2300 m.

90. Q. deliquescens C.H.Müll., Phytologia 42:289. 1979.

Distribución estatal en México: Chih. Altitud: 1700-2100 m.

91. Q. depressipes Trel., Mem.Natl.Acad.Sci. 20:90. 1924.

$=$ Q. bocoynensis C.H.Müll.; ${ }^{*} Q$. oblongifolia var. pallidinervis Trel.

Distribución estatal en México: Chih., Dgo. y Zac. También se distribuye en los EUA. Altitud: $2000-3000$ m.

92. Q. deserticola Trel., Mem.Natl.Acad.Sci. 20:79. 1924.

= Q. alveolata Trel.; Q. bipedalis Trel.; Q. deserticola var. incisa Trel.; Q. tamiapensis C.H.Müll.; Q. texcocana Trel.

Distribución estatal en México: DF, Gto., Hgo., Jal., Méx., Mich., Oax., Pue., Qro. y Sin. Altitud: $2000-2800$ m. 
93. Q. diversifolia Née, An.Ci.Nat. 3:270. 1801.

= Q. ambigua Humb. et Bonpl.; Q. bonplandiana Sweet.; Q. tomentosa var. diversifolia (Née) A.DC.

Distribución estatal en México: DF, Méx. y Pue.

94. Q. dumosa Nutt., N.Am.Sylv. 1:18. 1842.

= Q. dumosa f. crispata Trel.; Q. dumosa f. diversifolia Trel.; Q. dumosa f. insularis Trel.; Q. dumosa f. linearis Trel.; $Q$. dumosa f. longigemma Trel.; Q. dumosa f. microcarpa Trel.; Q. dumosa f. myrtifolia Trel.; Q. dumosa f. populifolia Trel.; Q. dumosa subsp. eudumosa A.Camus; Q. dumosa var. elegantula (Greene) Jeps.; Q. dumosa var. linearis (Trel.) A.Camus; Q. dumosa var. populifolia (Trel.) A.Camus; Q. $\times$ macdonaldii var. elegantyla Greene.

Distribución estatal en México: BC. También se distribuye en EUA. Altitud: 0-300 m.

95. Q. edwardsiae C.H.Müll., Am.Midl.Nat. 27:472. 1942.

Distribución estatal en México: NL.

96. Q. engelmanii Greene, III. W. Am.Oaks 1:32. 1889.

Distribución estatal en México: BC. También se distribuye en EUA. Altitud: 50-1200 m.

97. Q. filiformis C.H.Müll., Am.Midl.Nat. 27:473. 1942.

Distribución estatal en México: Coah.

98. Q. frutex Trel., Mem.Natl.Acad.Sci. 20:82, pl. 120. 1924.

Distribución estatal en México: DF, Hgo., Méx., Mich., Oax., Pue. y Tlax. Altitud: 2300-3000 m.

99. Q. fusiformis Small, FI.S.E.U.S.: 353. 1903.

= Q. oleoides var. quaterna C.H.Müll.; Q. virginiana var. fusiformis (Small) Sarg.; Q. virginiana var. macrophylla Sarg.; Quercus virginiana subsp. fusiformis (Small) E.Murray.

Distribución estatal en México: Coah., NL y Tamps. También se distribuye en EUA. Altitud: 0-1200 m.

100. Q. gambelli Nutt, J.Acad.Nat.Sci.Philadelphia, II, 1:179. 1847.

= Q. alba var. gunnisonii Torr. et A.Gray; Q. albifolia C.H.Müll.; Q. confusa Wooton et Standl.; Q. douglasii var. gambelii (Nutt.) A.DC.; Q. douglasii var. novomexicana A.DC.; Q. eastwoodiae Rydb.; Q. gambelii subsp. eugambelii A.Camus; Q. gambelii subsp. vreelandii (Rydb.) A.Camus; Q. gambelii var. bonina S.L.Welsh; Q. gambelii var. gunnisonii (Torr. et A.Gray) Wenz.; Q. gambelii var. novomexicana (A.DC.) Garrett; Q. gambelii var. utahensis (A.DC.) Garrett; Q. gambelii var. vreelandii (Rydb.) A.Camus; Q. gunnisonii Rydb.; Q. leptophylla Rydb.; Q. marshii C.H.Müll.; Q. media Wooton et Standl.; Q. nitescens Rydb.; Q. novomexicana (A.DC.) Rydb.; Q. novomexicana var. andrewsii Trel.; Q. novomexicana var. nitescens (Rydb.) A.Camus; Q. novomexicana var. typica A.Camus; Q. obtusifolia Rydb.; Q. pauciloba subsp. confusa (Wooton et Standl.) A.Camus; Q. stellata var. utahensis A.DC.; Q. submollis Rydb.; Q. subobtusifolia A.Camus; Q. undulata var. gambelii (Nutt.) Engelm.; Q. undulata var. gunnisonii (Torr. et A.Gray) Engelm.; Q. undulata var. obtusifolia A.DC.; Q. utahensis (A.DC.) Rydb. Q. utahensis subsp. euutahensis A.Camus; $Q$. utahensis var. mollis Sarg.; Q. utahensis var. submollis (Rydb.) Sarg.; Q. utahensis subsp. submollis (Rydb.) A.Camus; Q. vreelandii Rydb.

Distribución estatal en México: Chih., Coah. y Son. También se distribuye en EUA. Altitud: 1000-3000 m.

101. Q. germana Cham. et Schlecht., Linnaea 5:78. 1830.

$={ }^{*} Q$. galeotti M.Martens et Galeotti; Q. germana var. lemmoni Trel.; Q. substenocarpa Trel.

Distribución estatal en México: Hgo., Oax., Pue., SLP, Tamps. y Ver. Altitud: 800-1800 m.

102. Q. glabrescens Benth., Pl.Hartweg. 348:56. 1840.

= Q. glabrescens var. radlkoferiana (Trel.) A.Camus; Q. radlkoferiana Trel.

Distribución estatal en México: DF, Hgo., Méx., Mich., Oax., Pue., SLP, Tlax. y Ver. Altitud: 2450-3300 m.

103. Q. glaucescens Humb. et Bonpl. Pl.Aequinoct. 2(10):[25] 29. pl. 78. 1809.

= Q. cuneifolia Liebm.; Q. chinantlensis Liebm.; Q. nigrirhachis Trel.; Q. obscurirachis Trel.; Q. pinalensis Trel.; Q. synthetica Trel.; Q. texequitzinae Trel.

Distribución estatal en México: Gro., Jal., Mich., Nay., Oax., Sin. y Ver. Altitud: 250-1400 m.

104. Q. glaucoides M.Martens et Galeotti, Bull.Acad.Brux. 10, pt. 1:209. 1843. 
=Q. baldoquinae Trel.; Q. cancellata Trel.; Q. cancellata Trel.; Q. conjugens Trel.; Q. cordata M.Martens et Galeotti; Q. glaucophylla f. flacolulana Trel.; Q. glaucophylla f. lobata C.H.Müll.; Q. glaucophylla f. lobata C.H.Müll; Q. glaucophylla f. longifolia C.H.Müll; Q. glaucophylla f. macropetiolata C.H.Müll.; Q. glaucophylla Seemen; Q. glaucophylla var. subrotundifolia C.H.Müll.; Q. harmsiana Trel.; Q. mixtecana Trel.

Distribución estatal en México: Gro., Gto., Hgo., Jal., Méx., Mich., Mor., Nay., Oax., Pue., SLP, Sin. y Zac. Altitud: 500-2200 m.

105. Q. greggii (A.DC) Trel., Cont.U.S.Natl.Herb. 23(2):185. 1922.

= Q. loesneri Trel.; Q. greggii f. subglabra C.H.Müll.; Q. reticulata var. greggii A.DC. Q. derrumbaderoensis C.H.Müll.; Q. reticulata f. pungens C.H.Müll.

Distribución estatal en México: Coah., Dgo., Hgo., Nay., NL, Oax., Pue., SLP, Tamps. y Ver. Altitud: $2000-2900$ m.

106. Q. grisea Liebm., Overs.Dansk.Vidensk.Selsk. 171. 1854.

= Q. undulata Torrey var. grisea (Liebm.) Engelm.

Distribución estatal en México: Ags., Chih., Coah., Dgo., Gto., Jal., NL, SLP, Son., Ver. y Zac. También se distribuye en EUA. Altitud: $1500-2200 \mathrm{~m}$.

107. Q. hinckleyi C.H.Müll., Contr.Texas Res.Found., Bot.Stud. 1:40. 1951.

Distribución estatal en México: Chih. y Son. También se distribuye en EUA. Altitud: 1150-1500 m.

108. Q. ignasiensis C.H.Müll., Am.Midl.Nat. 27:475. 1942.

Distribución estatal en México: Son. Altitud: 920 m.

109. Q. insignis M.Martens et Galeotti, Bull.Acad.Brux. 10:219. 1843.

= Q. schippii St.; Q. tomentocaulis C.H.Müll.; Q. strombocarpa Liebm.; Q. insignis var. strombocarpoides Liebm.

Distribución estatal en México: Oax. y Ver. También se distribuye en CAm (Guatemala y Honduras). Altitud: 1500-2000 m.

110. Q. intricata Trel., Contr.U.S.Natl.Herb. 23:185. 1922.

= Q. intricata f. angusta Trel.; Q. intricata f. erratica Trel.; Q. intricata f. ovata Trel.; Q. intricata f. typica A.Camus; Q. microphylla var. crispata A.DC.

Distribución estatal en México: Chih., Coah., Dgo., NL y Zac. También se distribuye en EUA. Altitud: 1500-2500 m.

111. Q. invaginata Trel., Mem.Natl.Acad.Sci. 20:87. 1924.

= Q. invagintata f. purpusiana Trel.

Distribución estatal en México: Chih., Coah. y NL.

112. Q. jaralensis Trel., Mem.Natl.Acad.Sci. 20:86. 1924.

$=Q$. jaralensis f. berlandieri Trel.

Distribución estatal en México: Chih., Coah., Dgo. y Gto.

113. Q. laceyi Small, Bull.Torrey Bot.Club 28:358. 1901.

= Q. brevilova Sarg.; Q. brevilova subsp. laceyi (Small) A.Camus

Distribución estatal en México: Coah. y NL. También se distribuye en EUA. Altitud: 350-2200 m.

114. Q. laeta Liebm., Overs.Dansk.Vidensk.Selsk. 179. 1854.

=Q. centralis Trel.; Q. centralis f. acherdophylla Trel.; Q. laeta f. heterophylla Trel.; Q. obscura Trel.; Q. pallescens Trel.; Q. prinopsis Trel.; Q. transmontana Trel.; Q. transmontana var. obscura (Trel.) A.Camus; Q. obscura var. perpusilla Trel.

Distribución estatal en México: Ags., Coah., DF, Dgo., Gto., Hgo., Jal., Méx., Mich., Nay., NL, SLP, Sin., Oax. y Zac. Altitud: $1900-2700 \mathrm{~m}$.

115. Q. lancifolia Cham. et Schltdl., Linnaea 5:78 (1830).

= Q. lancifolia f. pilosiuscula Wenz.; Q. boqueronae Trel.

Distribución estatal en México: Chis. y Ver. También se distribuye en CAm (Guatemala).

116. Q. laxa Liebm., Overs.Dansk.Vidensk.Selsk. 181. 1854.

=Q. callosa M.Martens ex A.DC.; Q. reticulata laxa Wenz.; Q. xylina Scheidw.

Distribución estatal en México: Col., Jal., Mich., Nay. y Sin. 
117. Q. leiophylla A.DC. in A.P. de Candolle, Prodr. 16(2):71. 1864.

$={ }^{*}$ Q. excelsa Liebm.; Q. lancifolia var. monocarpa Wenz.; Q. leiophylla f. subintegra Trel.; Q. toxicodendrifolia Trel.

Distribución estatal en México: Hgo., Oax., Pue. y Ver. Altitud: 1950-3000 m.

118. Q. liebmanii Oerst., Chênes Amér.Trop., p. 16, pl. I, 33, 1869.

$={ }^{*} Q$. poculifer Trel.; ${ }^{*} Q$. liebmanii f. brevipes Trel.

Distribución estatal en México: Gro., Oax. y Pue. Altitud: 1000-2450 m.

119. Q. macdougalli M.Martínez, An.Inst.Biol.Univ.Nac.Aut.México 34:147. 1964.

Distribución estatal en México: Oax. Altitud: 2600-2900 m.

120. Q. magnoliifolia Née, An.Ci.Nat. 268. 1801.

= Q. circinata Née; Q. erubescens Trel.; Q. flava Née ex Spreng.; Q. haematophlebia Trel.; Q. lutea Née; Q. macrophylla Née; Q. magnoliifolia var. lutea (Née) A.DC.; Q. magnoliifolia var. macrophylla (Née) A.DC.; Q. neoplatyphylla A.Camus; Q. nudinervis Liebm.; Q. platyphylla E.F. Warb.; Q. rubescens Trel.; Q. tepicana Trel.

Distribución estatal en México: Col., Gro., Hgo., Jal., Méx., Mich., Mor., Nay., Oax., Pue. y Sin. Altitud: 170-2900 m.

121. Q. martinezii C.H.Müll., An.Inst.Biol.Univ.Nac.Aut.México 24:274. 1953.

Distribución estatal en México: Gro., Jal., Méx., Mich., Nay. y Oax. Altitud: 1800-2600 m.

122. Q. microphylla Née, An.Ci.Nat. 3:264. 1801.

$=$ Q. frutex var. uhdeana Trel.; Q. microphylla var. uhdeana (Trel.) A.Camus.

Distribución estatal en México: Ags., Coah., Gto., Hgo., Jal., Nay., NL, Oax., Pue., Qro., SLP, Tamps. y Ver. Altitud: 2000-2500 m.

123. Q. mohoriana Bluck ex Rydb., Bull.N.Y.Bot.Gard. 2:219. 1901.

Distribución estatal en México: Coah. También se desarrolla en EUA. Altitud: 600-2500 m.

124. Q. monterreyensis Trel. et Mull., Bull.Torrey Bot.Club 63:151. 1936.

Distribución estatal en México: NL. Altitud: $600-1300 \mathrm{~m}$.

125. Q. muehlenbergii Englem, Trans.Acad.Sci.St.Louis 3:391. 1877.

= Q. acuminata (Michx.) Sarg.; Q. acuminata var. alexandrii Farw.; Q. alexanderi Britton; Q. brayi Small; Q. castanea Muhl.; Q. castanea var. macrophylla Hampton; Q. muehlenbegii Brayi (Small); Q. muehlenbergii f. alexanderi (Britton) Trel.; Q. muehlenbergii var. brayi (Small) Sarg.; Q. muehlengergii var. alexanderi (Britton) Steyerm.; Q. prinoides var. acumianta (Michx.) Gleason; Q. prinus Coulter; Q. prinus var. acuminata Michx.; Q. rubra var. muehlenbergii (Engelm.) Wenz.; Q. sentenelensis C.H.Müll.

Distribución estatal en México: Coah., NL, Tamps. y Ver. También se distribuye en EUA. Altitud: 0-2300 m.

126. Q. oblongifolia Torr. in R.B.Marcy, Explor.Red River Louisiana: 173. 1853.

Distribución estatal en México: Chih., Coah., Dgo. y Son. También se distribuye en EUA. Altitud: 1300-1650 m.

127. Q. obtusata Humb. et Bonpl. PI.Aequinoct. 2(10):26. 1809.

= Q. alvarezensis Trel.; Q. atriglans E. F. Warb.; Q. crenatifolia Trel.; Q. hartwegii Benth.; Q. hartwegii f. glabrata Trel.; Q. obtusata var. hartwegii (Benth) A.DC.; Q. obtusata var. pandurata (Bonpl.) A.DC.; Q. obtusata var. typica A.Camus; Q. pandurata Bonpl.; Q. pandurata var. hartwegii (Benth.) Wenz.; Q. panduriformis f. colimensis Trel.; Q. panduriformis f. rubrinervis Trel.; Q. panduriformis Trel.; Q. rugosa sensu Trel.; Q. spicata Humb. et Bonpl.

Distribución estatal en México: DF, Dgo., Gro., Gto., Hgo., Jal., Méx., Mich., Mor., Nay., NL, Oax., Pue., Qro., SLP, Qro., Ver. y Zac. Altitud: 620-2580 m.

128. Q. oleoides Schltdl. et Cham., Pl.Mexicanum. Linnaea 5:79. 1830.

= Q. lutescens M.Martens et Galeotti; Q. oleoides f. lutescens Trel.; Q. oleoides var. australis Trel.; Q. oleoides var. typica A.Camus; $Q$. retusa Liebm.

Distribución estatal en México: Cam., Chis., Hgo., Oax., Pue., SLP, Tab., Tamps., Ver. y Yuc. También se distribuye en CAm (hasta Costa Rica). Altitud: 150-960 m.

129. Q. oocarpa Liebm., Overs.Dansk.Vidensk.Selsk. 184. 1854. 
= Q. glabrescens Seemen; Q. insignis subsp. oocarpa (Liebm.) E.Murray; Q. insignis var. oocarpa (Liebm.) E.Murray; Q. warszewiczii Liebm.; Q. yunckeri Trel.

Distribución estatal en México: Gro., Jal. y Nay. También se distribuye en CAm (Guatemala, Costa Rica y Panamá). Altitud: $1400-2000 \mathrm{~m}$.

130. Q. opaca Trel., Mem.Natl.Acad.Sci. 20:92. 1924.

Distribución estatal en México: Méx., Hgo., Oax. y Tamps.

131. Q. × pastorensis C.H.Müll., J.Arn.Arbor. 17:164. 1936. (Q. clivicola Trel. et C.H.Müll. × Q. glaucoides M.Martens et Galeotti).

Distribución estatal en México: NL. Altitud: ca. 1500 m.

132. Q. peduncularis Née, An.Ci.Nat. 3:270. 1801.

Q. achoteana var. sublanosa Trel. ex Yunck.; Q. affinis M.Martens et Galeotti; Q. aguanana Trel.; Q. ahoteana Trel.; Q. arachnoidea Trel.; Q. barbanthera Trel.; Q. barbanthera var. calva Trel.; Q. berbeyana Trel.; Q. callosa Benth.; $Q$. dolichopus E.F.Warb.;Q. martensiana Trel.; Q. peduncularis f. macrodonta (Trel.) A.Camus; Q. peduncularis subsp. calIosa A.Camus; Q. peduncularis subsp. eupeduncularis A.Camus; Q. peduncularis subsp. hurteri (Trel.) A.Camus; Q. peduncularis subsp. pilicuaulis (Trel.) A.Camus; Q. peduncularis var. obovalis (Trel.) A.Camus; Q. peduncularis var. sublanosa (Trel. ex Yunck.) C.H.Müll.; Q. peduncularis var. typica A.Camus; Q. pilicaulis f. concava C.H.Müll.; Q. pilicaulis f. elongata C.H.Müll.; Q. pilicaulis Trel.; Q. pilicuaulis f. armata Trel.; Q. pilicaulis f. exserta C.H.Müll.; Q. pilicaulis f. macrodonta Trel.; Q. pilicaulis f. obovalis Trel.; Q. tomentosa var. abbreviata A.DC.; Q. tomentosa var. bullata A.DC.; Q. tomentosa var. communis A.DC.; Q. tomentosa Willd.

Distribución estatal en México: Chis., Col., Gro., Jal., Méx., Mich. Nay., Oax., Pue. y Ver. También se distribuye en CAm (Guatemala, Belice, Honduras). Altitud: 340-1940 m.

133. Q. perpallida Trel., Mem.Natl.Acad.Sci. 20:91. 1924.

Distribución estatal en México: BC., Chih. y Son. Altitud: 1150-1650 m.

134. Q. pilarius Trel., Mem.Natl.Acad.Sci. 20:44. 1924.

$=$ Q. inignis var. pilarius (Trel.) E.Murray; Q. insignis subsp. pilarius (Trel.) E.Murray; Q. yousei Trel.

Distribución estatal en México: Chis. y Ver. También se encuentra en CAm (Honduras, Guatemala, Costa Rica). Altitud: 1150-2400 m.

135. Q. polymorpha Schltdl. et Cham., Linnaea 5:78. 1830.

= Q. guatimalensis A.DC. (pro parte); Q. petiolaris Benth.; Q. polymorpha f. angustifolia C.H.Müll.; Q. turbinata Liebm.; $Q$. varians M.Martens et Galeotti.

Distribución estatal en México: Chis., Hgo., NL, Oax., Pue., SLP, Tamps. y Ver. También se encuentra en EUA y CAm (Guatemala). Altitud: 2000-2450 m.

136. Q. potosina Trel., Mem.Natl.Acad.Sci. 20:84. 1924.

= Q. potosina f. aptera Trel.; Q. potosina f. exilis Trel.

Distribución estatal en México: Ags., Chih., Dgo., Gto., Jal., SLP y Zac. Altitud: 2000-2450 m.

137. Q. praeco Trel., Mem.Natl.Acad.Sci. 20:88. 1924.

Distribución estatal en México: Jal., Nay. y Zac. Altitud: 1700-2100 m.

138. Q. pringlei Seemen ex Loes., Bot.Jahrb.Syst. 29:96. 1900.

= Q. pringlei f. dentata C.H.Müll.

Distribución estatal en México: Coah., NL y Zac. Altitud: 1500-2300 m.

139. Q. pungens Liebm., Overs.Dansk.Vidensk.Selsk. 171. 1854.

=Q. undulata var. pungens (Liebm.) Engelm.; Q. undulata var. wrightii Engelm.

Distribución estatal en México: Chih. y Coah. También se encuentra en EUA. Altitud: 800-2000 m.

140. Q. repanda Humb. et Bonpl., Pl.Aequinoct. 2:31-32. pl. 79. 1809.

= Q. lecomteana Trel.; Q. revoluta Trel.; Q. subtriloba Trel.

Distribución estatal en México: Hgo., Pue. y Tlax. Altitud: 1700-2800 m. 
141. Q. resinosa Liebm., Overs.Dansk.Vidensk.Selsk. 182. 1854.

= Q. macrophylla sensu Trel. (no Q. macrophylla Née).

Distribución estatal en México: Ags., Dgo., Gto., Jal., Mich., Nay., SLP y Zac. Altitud: 1300- 2500 m.

142. Q. rugosa Née, An.Ci.Nat. 3:275. 1801.

Q. ariaefolia Trel.; Q. conglomerata Trel.; Q. decipiens M.Martens et Galeotti; Q. diversicolor Trel.; Q. diversicolor var. mearnsii Trel.; $Q$. diversicolor var. socorronis Trel.; Q. durangensis Trel.; Q. durangensis Trel.; Q. innuncupata Trel.; $Q$. macrophylla var. rugosa (Née) Wenz.; Q. purpusii Trel.; Q. reticulata f. applanata (Trel.) A.Camus; Q. reticulata f. concava (Trel.) A.Camus; Q. reticulata f. crenata (Trel.) A.Camus; Q. reticulata f. dugesii Trel.; Q. reticulata f. Ionga Trel.; Q. reticulata f. pungens C.H.Müll.; Q. reticulata Humb. et Bonpl.; Q. reticulata subsp. rhodophlebia (Trel.) A.Camus; Q. reticulata var. conglomerata (Trel.) A.Camus; $Q$. reticulata var. squarrosa Trel.; $Q$. reticulata var. typica A.Camus; $Q$. rhodophlebia f. applanata Trel.; Q. rhodophlebia f. typica A.Camus; Q. rhodophlebia Trel.; Q. rodophlebia f. concava Trel.; Q. rodophlebia f. crenata Trel.; Q. suchiensis E.F.Warb.; Q. uhdeana Trel.; Q. vellifera Trel.; Q. vellifera Trel.

Distribución estatal en México: Ags., Chis., Coah, Col., DF, Gro., Gto., Dgo., Hgo., Jal., Méx., Mich., Mor., NL, Oax., Pue., Qro., SLP, Son., Ver. y Zac. También se distribuye en EUA. Altitud: 1800-2900 m.

143. Q. sebifera Trel., Mem.Natl.Acad.Sci. 20:93. 1924.

= Q. ceripes Trel.; Q. schenckiana Trel.; Q. sebifera f. comitanensis Trel.; Q. trinidadensis C.H.Müll.

Distribución estatal en México: Chis., Hgo., NL, Oax., Pue., SLP, Tamps. y Ver. Altitud: 1600-2400 m.

144. Q. segoviensis Liebm., Overs.Dansk.Vidensk.Selsk. 186. 1854.

$=Q$. reticulata var. segoviensis (Liebm.) Wenz.

Distribución estatal en México: Chis. También se localiza en CAm (Guatemala, Honduras, Nicaragua). Altitud: 760$2500 \mathrm{~m}$.

145. Q. sinuata Walt., Fl.Carol. 235. 1788.

= Q. annulata Buckley; Q. breviloba (Torr.) Sarg.; Q. breviloba f. argentea Trel.; Q. breviloba subsp. eubreviloba A.Camus; Q. breviloba subsp. pseudocrispata (A.Camus) A.Camus; Q. durandii Buckley; Q. durandii var. breviloba (Torr.) E.J.Palmer; Q. durandii var. sansabeana (Buckley) Buckley; Q. obtusifolia var. breviloba Torr.; Q. pseudocrispata A.Camus; Q. sansabeana Buckley; Q. sinuata subsp. breviloba (Torr.) E.Murray; Q. sinuata var. breviloba (Torr.) C.H.Mül.; Q. undulata var. breviloba (Torr.) Engelm.

Distribución estatal en México: Coah., NL y Tamps. También se localiza en EUA. Altitud: 200-600 m.

146. Q. splendens Née, An.Ci.Nat. 3:275. 1801.

$=Q$. sororia Liebm.; $Q$. crassifolia Benth.

Distribución estatal en México: Gro., Jal., Mich., Nay. y Oax. Altitud: 1000-2300 m.

147. Q. striatula Trel., Mem.Natl.Acad.Sci. 20:93. 1924.

Distribución estatal en México: Chih., Dgo., Zac. Altitud: 2100-3000 m.

148. Q. subspathulata Trel., Mem.Natl.Acad.Sci. 20:56. 1924.

$=$ Q. pallidifolia C.H.Müll.

Distribución estatal en México: Chih., Jal. y Son. Altitud: 1500-1600 m.

149. Q. tinkhamii C.H.Müll., Am.Midl.Nat. 27:481. 1942.

Distribución estatal en México: NL y SLP.

150. Q. toumeyi Sarg., Gard.Forest 8:92. 1895.

= Q. chuichupensis C.H.Müll.; Q. hartmanii Trel.

Distribución estatal en México: Chih. y Son. También crece en EUA. Altitud: 1500-2400 m.

151. Q. tuberculata Liebm., Overs.Dansk.Vidensk.Selsk. 181. 1854.

= Q. aurantiaca Trel.; Q. idonea Goldman; Q. standleyi Trel.; Q. tuberculata f. latifolia M.Martínez.

Distribución estatal en México: BC, Chih., Dgo., Mich., Nay., Sin. y Son. Altitud: 900-1700 m.

152. Q. turbinella Greene, III.W.Amer.Oaks 1:37. 1889. 
DiVERSIDAD DEL GÉNERO QUERCUS EN MÉXICO

=Q. dumosa subsp. turbinella (Greene) E.Murray; Q. dumosa var. turbinella (Greene) Jeps.; Q. subturbinella Trel.

Distribución estatal en México: BC, Chih. y Son. También se desarrolla en EUA. Altitud: 800-2000 m.

153. Q. undata Trel., Mem.Natl.Acad.Sci. 20:86. 1924.

Distribución estatal en México: Dgo. Altitud: ca. 2500 m.

154. Q. × undulata Torr. pro spp., Ann.Lyceum Nat.Hist.New York 2:248. 1828.

= Q. lesueuri C.H.Müll.; Q. pauciloba Rydb.; Q. undulata var. jamesii Engelm.; Q. undulata var. pedunculata A.DC.; Q. undulata var. rydbergiana Cockerell; $Q$. undulata var. typica A.Camus; $Q . \times$ fendleri Liebm.; Q.× rydbergiana Cockerell; Q. $\times$ venustula Greene.

Distribución estatal en México: Chih. También crece en EUA.

155. Q. vaseyana Buckley, Bull.Torrey Bot.Club 10:91. 1883.

=Q. pungens subsp. vaseyana (Buckley) E.Murray; Q. pungens var. vaseyana (Buckley) C.H.Müll.; Q. sillae Trel.; Q. undulata var. vaseyana (Buckley) Rydb.

Distribución estatal en México: Chih., Coah. y NL. También se distribuye en EUA. Altitud: 300-1500 m.

156. Q. verde C.H.Müll., J.Arn.Arbor. 17:163. 1936.

Distribución estatal en México: NL. Altitud: 2100-2500 m.

157. Q. vicentesis Trel., Mem.Natl.Acad.Sci. 20:59. 1924.

=Q. comasaguana Trel.; Q. siltepecana Matuda

Distribución estatal en México: Chis. También se distribuye en CAm (Guatemala, El Salvador). Altitud: 1200-1900 m.

C. Sección Protobalanus (Trel.) A.Camus (encinos intermedios, o encinos de copa dorada)

158. Q. cedrosensis C.H.Müll., Madroño, 16:186-192. 1962.

Distribución estatal en México: BC. Altitud: $1200 \mathrm{~m}$.

159. Q. chrysolepis Liebm., Overs.Dansk.Vidensk.Selsk. 173. 1854.

= Q. chrysophyllus Kellogg; Q. chrysolepis f. grandis Jeps.; Q. chrysolepis var. grandis (Jeps.) Jeps.; Q. chrysolepis f. hansenii Jeps.; Q. chrysolepis var. hansenii (Jeps.) Jeps.; Q. chrysolepis f. nana Jeps.; Q. chrysolepis var. nana (Jeps.) Jeps.; Q. chrysolepis f. pendula Jeps.; Q. chrysolepis var. pendula (Jeps.) Jeps.; Q. crassipocula Torr.; Q. fuvescens Kellogg, Q. oblongifolia R.Br.Ter.; Q. oblongifolia var. pallidinervis Trel.; Q. wilcoxii Rydb; Q. chrysolepis var. wilcoxii (Rdb.) E.Murray. Q. chrysolepis subsp. wilcoxii (Rydb.) E.Murray.

Distribución estatal en México: BC y Chih. También se localiza en EUA. Altitud: 200-2600 m.

160. Q. palmeri Engl., S.Watson, Bot.California 2:97. 1880.

$=Q$. chrysolepis var. palmeri (Engelm.) Engelm.; Q. dunni Kellogg ex Curran.

Distribución estatal en México: BC. También se distribuye en EUA. Altitud: 700-1800 m.

161. Q. tomentella Engelm., Trans.Acad.Sci.St.Louis, 3:393. 1877.

Q. chrysolepis var. tomentella (Engelm.) E.Murray; Q. chrysolepis subsp. tomentella (Engelm.) E.Murray; Q. tomentella var. conjungens Trel. Q. tomentella f. conjugens (Trel.) Trel.

Distribución estatal en México: BC, Isla Guadalupe. También se desarrolla en EUA. Altitud: 100-650 m. 